\title{
Biomarkers of Vascular Injury and Type 2 Diabetes: A Prospective Study, Systematic Review and Meta-Analysis
}

\author{
Laura Pletsch-Borba ${ }^{1,2, *}$, Cora Watzinger ${ }^{1}$, Renée Turzanski Fortner ${ }^{1}$, Verena Katzke ${ }^{1}$, \\ Lukas Schwingshackl $^{3,4}$, Solomon A. Sowah 1,2 ${ }^{10}$, Anika Hüsing ${ }^{1}$, Theron Johnson ${ }^{1}$, \\ Marie-Luise Groß ${ }^{5}$, Sandra González Maldonado ${ }^{1}$, Michael Hoffmeister ${ }^{6}$, Peter Bugert ${ }^{7,8}$, \\ Rudolf Kaaks ${ }^{1}$, Mirja Grafetstätter ${ }^{1,2}$ and Tilman Kühn ${ }^{1}$ (D) \\ 1 Division of Cancer Epidemiology, German Cancer Research Center (DKFZ), Im Neuenheimer Feld 581, \\ 69120 Heidelberg, Germany; cora.watzinger@med.uni-muenchen.de (C.W.); r.fortner@dkfz.de (R.T.F.); \\ v.katzke@dkfz.de (V.K.); s.sowah@dkfz.de (S.A.S.); a.huesing@dkfz.de (A.H.); t.johnson@dkfz.de (T.J.); \\ s.gonzalezmaldonado@dkfz.de (S.G.M.); r.kaaks@dkfz.de (R.K.); m.grafetstaetter@dkfz.de (M.G.); \\ t.kuehn@dkfz.de (T.K.) \\ 2 Faculty of Medicine, University Medicine Heidelberg, Im Neuenheimer Feld 672, \\ 69120 Heidelberg, Germany \\ 3 Institute for Evidence in Medicine, Medical Center - University of Freiburg, Breisacher Straße 153, \\ 79110 Freiburg, Germany; shwingshackl@cochrane.de \\ 4 Faculty of Medicine, University of Freiburg, Breisacher Straße 153, 79110 Freiburg, Germany \\ 5 Department of Preventive Oncology, National Centre for Tumor Diseases, Im Neuenheimer Feld 460, \\ 69120 Heidelberg, Germany; marie-luise.gross@nct-heidelberg.de \\ 6 Division of Clinical Epidemiology and Aging Research, German Cancer Research Center (DKFZ), Im \\ Neuenheimer Feld 581, 69120 Heidelberg, Germany; m.hoffmeister@dkfz.de \\ 7 Institute of Transfusion Medicine and Immunology, University Medicine Heidelberg, Friedrich-Ebert-Straße \\ 107, 68167 Mannheim, Germany; peter.bugert@medma.uni-heidelberg.de \\ 8 German Red Cross Blood Service Baden-Württemberg-Hessen, Friedrich-Ebert-Straße 107, \\ D-68167 Mannheim, Germany \\ * Correspondence: 1.pletschborba@dkfz.de; Tel.: +49-6221-42-2352
}

Received: 28 October 2019; Accepted: 25 November 2019; Published: 27 November 2019

\begin{abstract}
Data on biomarkers of vascular injury and type 2 diabetes (T2D) risk from prospective studies are lacking. We evaluated seven biomarkers of vascular injury in relation to T2D. Additionally, a meta-analysis was performed. From the EPIC-Heidelberg cohort, 2224 participants were followed-up from baseline for 16 (median) years. E-Selectin, P-Selectin, intercellular adhesion molecule 3 (ICAM3), thrombomodulin, thrombopoietin, glycoprotein IIb/IIIa and fibrinogen levels were measured in baseline blood samples. The systematic review and meta-analysis included prospective studies identified through MEDLINE and Web of Science that investigated the association between mentioned biomarkers and T2D. The study population included 55\% women, median age was 50 years, and 163 developed T2D. ICAM3 was associated with lower T2D risk (fully adjusted $\mathrm{HR}_{\text {highest vs. lowest }}$ tertile 0.62 (95\% CI: $0.43,0.91)$ ), but no other studies on ICAM3 were identified. Overall, fifteen studies were included in the systematic review and meta-analysis $(6,171$ cases). E-Selectin was associated with higher T2D risk HR $\mathrm{per} \mathrm{SD}_{1}: 1.34$ (95\% CI: 1.16, 1.54; $\mathrm{I}^{2}=63 \%, n=9$ studies), while thrombomodulin was associated with lower risk $\mathrm{HR}_{\text {per SD: }} 0.82$ (95\% CI: $0.71,0.95 ; \mathrm{I}^{2}=0 \%, n=2$ studies). In the EPIC-Heidelberg, ICAM3 was associated with lower T2D risk. The meta-analysis showed a consistent positive association between E-Selectin and T2D. It was also suggestive of an inverse association between thrombomodulin and T2D, although further studies are needed to corroborate this finding.
\end{abstract}


Keywords: Epidemiology; Type 2 Diabetes; E-Selectin; P-Selectin; ICAM3; thrombomodulin; vascular injury biomarkers

\section{Introduction}

An estimated $8 \%$ of the world's adult population live with type 2 diabetes mellitus (T2D), with the global prevalence predicted to increase in the coming years [1]. According to the Global Burden of Disease study, diabetes was the 12th leading cause of death, and total deaths from T2D increased from 2007 to 2017 by nearly 50\% [2]. Microvascular dysfunction has been suggested to be one mechanistic pathway linking obesity to increased insulin resistance and, subsequently, T2D [3-6]. Endothelial dysfunction and insulin resistance are closely related phenomena, as it has been proposed that trans-capillary insulin transport is a rate-limiting step in peripheral insulin action [7]. Endothelial dysfunction can also diminish insulin's delivery to the interstitium and could thereby limit insulin action [7]. In this context, vascular injury could be a risk factor for T2D.

The endothelium is a widely distributed organ system and plays an important role in both maintaining blood in its fluid state, as well as in providing controlled haemostasis at sites of vascular injury [8]. Vascular injury can be assessed by circulating concentrations of biomarkers related to inflammation, haemostasis and endothelial activation [4]. Major markers of vascular injury include soluble E-Selectin and P-Selectin, intercellular adhesion molecule 3 (ICAM3), thrombomodulin, thrombopoietin, glycoprotein IIb/IIIa (GP IIb/IIIa) and fibrinogen. E-Selectin is expressed in endothelial cells and is known to be responsible for the neutrophil-rolling interaction with the endothelium [9]. P-Selectin is stored in the Weibel-Palade bodies of the alpha-granules of platelets and is expressed in endothelial cells [10-12]. ICAM3 may mediate interactions between leucocytes and endothelial cells in atherosclerosis, becoming functionally altered during apoptosis to mediate clearance of apoptotic leucocytes through suggested generation of "eat me" signals [13-15]. Thrombomodulin is a membrane glycoprotein expressed by endothelial cells of all vessels (arteries, veins, capillaries and lymphatics) that converts thrombin from a procoagulant to an anticoagulant enzyme thus modulating secondary haemostasis $[16,17]$. Thrombopoietin is a humoral growth factor produced by the liver and kidney and is the most important growth factor in the regulation of megakaryocytes development and platelet synthesis [18-21]. GP IIb/IIIa is a receptor expressed on the surface of activated platelets, essential for platelet aggregation via binding to fibrinogen [22].

We have previously evaluated the mentioned biomarkers in relation to cardiovascular risk factors and myocardial infarction (MI) risk in a population-based study. While we did not observe prospective associations with MI, there were several associations with cardiovascular risk factors [23]. For example, plasma levels of P-Selectin, E-Selectin and ICAM3 were higher among study participants with prevalent diabetes. Moreover, thrombomodulin has been shown to be inversely associated with T2D in the population-based MONICA/KORA study [24], and further previous studies indicate that E-Selectin levels may be associated with increased diabetes risk [4,5,25-30]. Considering the biological properties of the mentioned biomarkers, our own analyses on cardiovascular risk factors, and previous findings from other population-based studies, we decided to evaluate the aforementioned biomarkers in relation to T2D risk in the population-based EPIC-Heidelberg study. Hypothesizing that vascular injury, measured through circulating biomarkers, could be a risk factor for T2D, we studied the associations between the biomarkers of vascular injury and incidence of T2D in a subcohort EPIC-Heidelberg including women and men aged 35-65 at baseline, who were followed-up for 16 years. We also performed a systematic review on the evidence for associations between these markers and incident T2D, and a meta-analysis in order facilitate the contextualization of our results. 


\section{Materials and Methods}

\subsection{Study Population (Original Research)}

The EPIC-Heidelberg cohort included 25,540 participants aged 35-65 years, who were recruited between 1994 and 1998 from the general population of Heidelberg, Germany and the surrounding areas [31,32]. At baseline, participants completed detailed questionnaire-based and interviewer-administered assessments on diet, lifestyle factors, medication use and health status, and anthropometric measurements were taken. Blood samples were collected and processed using a standardized protocol, and have been stored in gas phase liquid nitrogen at $-150^{\circ}$ Celsius. Details of blood sample collection and processing have been previously described in detail [32]. The present analyses on plasma biomarkers and T2D risk were conducted using a randomly selected subcohort of approximately $10 \%$ of the original cohort. This subcohort was initially drawn for case-cohort analyses on biomarkers in relation to risks of common cancers and cardiovascular diseases [33,34] and included 2224 EPIC-Heidelberg participants free of diabetes at baseline ( $n=194$ prevalent cases excluded). Follow-up was conducted through 31st December 2012. The Ethics Committee of the Heidelberg University Hospital approved the study and all participants gave written informed consent.

\subsection{Ascertainment of Incident Diabetes Mellitus (Original Research)}

Participants have been followed through a combination of active and passive follow-up methods since baseline [31], and completed follow-up questionnaires at 2-3 year intervals following recruitment, with response rates of circa 95\% [35]. Incident cases of T2D were identified based on self-report of a new diagnosis of diabetes, use of diabetes relevant medication, or change in diet due to disease. Self-reported cases were validated by a trained study physician using diagnostic records obtained from the treating physician. In addition to diagnoses reported on questionnaires, information from death certificates and record linkage with the major hospital in the area were conducted, again followed by a validation based on individual records.

\subsection{Laboratory Methods (Original Research)}

Details on the laboratory methods have been previously described [23]. Briefly, E-Selectin, P-Selectin, ICAM3 and thrombomodulin were measured with the "Quiplex SQ 120" instrument from Meso Scale Discoveries (MSD Maryland, USA) using the "human vascular injury kit I" multiplex assay kit. Thrombopoietin was measured by electrochemoluminescence immunoassays (ECLIA) ("U-Plex TPO Assay" kit from MSD). Enzyme-linked immunosorbent assays (ELISA) was used to measure GP IIb/IIIa and fibrinogen levels, using the essay kits "ab108851" from Abcam (Cambridge, UK) and "KA0475" from Abnova (Heidelberg, Germany). Within- and between-batch coefficients of variation were as follows (within-batch CV (between-batch CV)): P-Selectin 3.3\% (9.1\%), E-Selectin 3.6\% (10.6\%), thrombomodulin 3.8\% (10.1\%), thrombopoietin 4.6\% (19.5\%), GP Ilb/IIIa 5.5\% (46.9\%) and ICAM3 7.5\% (10.2\%) (Supplementary Table S4) [33]. All biomarkers showed good biological reproducibility in a pilot study carried out prior to the present analyses including 78 participants from the EPIC-Heidelberg subcohort, with Spearman's coefficients $(\rho)$ for intra-individual correlations over one year of 0.88 (E-Selectin), 0.80 (P-Selectin), 0.69 (ICAM3), 0.63 (thrombomodulin), 0.73 (thrombopoietin), and 0.51 (GP IIb/IIIa) (Supplementary Table S4) [23,36]. Biological reproducibility of fibrinogen was not assessed, as exhaustive previous repeatability analyses indicated low intra-individual variation over time [37]. The batch mean-centering method was used for batch standardization [38]. Inter-correlations among the measured biomarkers were mild $(\rho<0.4)$, except for E-Selectin and P-Selectin $(\rho=0.56)[23]$.

\subsection{Covariates Assessment}

Covariates assessment is described in detail in the Supplementary Material. 


\subsection{Systematic Review and Meta-Analysis}

We searched MEDLINE and Web of Science databases following PRISMA guidelines [39] (through 4th June 2019), without language restriction, using the search strategies provided in the Supplementary Material. Titles, abstracts and full-texts were screened independently by 2 reviewers in the first step (LPB and CW). As double check, 2 reviewers have each re-screened half of the total list of titles (SAS and TK). In case of disagreement between the reviewers, consensus was reached through discussion. Inclusion criteria: (i) Population-based prospective cohort, case-cohort and nested case-control studies; (ii) that evaluated circulating concentrations of at least one of the 7 aforementioned vascular dysfunction biomarkers; (iii) in participants free of T2D at baseline. We examined the reference lists of the selected papers to find additional relevant articles. In cases where potentially relevant results were referred to as "unpublished data" in reviewed full-text articles, we contacted the study authors. The following data was extracted from the full texts: study design, baseline characteristics of the study population, number of participants without T2D at baseline, incident cases of T2D, mean follow-up, definition of T2D, crude and adjusted odds ratios, relative risks or HRs with respective $95 \%$ confidence intervals (CI) and adjusting covariates included in the multivariate analyses (Tables 3 and 4). Quality of the studies was assessed using the adapted Newcastle-Ottawa Scale (Supplementary Tables S1 and S2) [40]. International Prospective Register of Systematic Reviews PROSPERO 2019: CRD42019125922.

\subsection{Statistical Analyses}

Continuous variables are described as median (percentile25; percentile75) and categorical variables as numbers (percentages). T-Tests (for continuous variables) and Chi-square (for categorical variables) tests were used for calculation of $p$-values for differences in covariates between T2D cases and non-cases. Values of all plasma biomarkers were $\log 10$ transformed so that the distribution better approximated a normal distribution, and batch-standardized (mean of 0 and standard deviation SD of 1). We categorized biomarker levels by tertiles using thresholds based on sex-specific distributions in the cohort, as biomarker levels were generally higher in samples of men compared to those of women. Percentage of missing values in the covariates was $8 \%$ (Supplementary Table S3). Missing values were multiply imputed under the missing at random assumption, using a fully conditional algorithm, with number of imputations set to 5 ; results from the 5 imputed datasets were pooled using SAS procedure PROC MIANALYZE.

We used Cox proportional hazards regression models to calculate hazard ratios (HRs) and 95\% confidence intervals (CI) [41]. Each plasma biomarker was analysed as an individual risk factor for incident T2D. We used age as a time scale, and all participants were left-truncated at the time of blood sampling, and censored at T2D diagnosis, loss to follow-up, or end of the study follow-up, whichever occurred first. Model 1 was adjusted for age and sex. Model 2 was additionally adjusted for body mass index (BMI) $\left(\mathrm{kg} / \mathrm{m}^{2}\right)$, alcohol consumption (g/day in the past year), smoking status (never, past quit $\geq 10$ years ago, past quit $<10$ years ago, current $<15$ cigarettes/day, current $\geq 15$ cigarettes/day), physical activity (Cambridge index, inactive/moderately inactive vs. moderately active/active), education level (primary, secondary and university), self-reported hypertension (yes/no), glycated haemoglobin $(\mathrm{HbA} 1 \mathrm{c})$ and C-Reactive protein (CRP). All covariates were determined a priori. Further adjustments for fibre intake, meat consumption, and coffee intake were additionally performed in sensitivity analyses. Extended correlation tests based on Schoenfield residuals indicated no violations of the proportional hazards assumption. Potential effect modifications of sex and age were tested including a multiplicative term into the Cox regression models. Non-linear associations between the biomarkers and T2D risk were tested using restricted natural splines, and the best fitting regression model was chosen based on the lowest Akaike Information Criteria. Additionally, we estimated to which extent the vascular injury biomarkers improve the prediction of absolute T2D risk beyond the covariates mentioned above using the concordance (C-) statistic, also known as area under the ROC curve (AUROC), calculated by logistic regression analyses. Our basic model for risk prediction included all the variables used as covariates from multivariable Cox regression analyses. Biomarkers were then 
added to the basic model to evaluate changes in the AUROC. The improvement in discriminative capacity in terms of $\mathrm{C}$-statistics between nested models with and without biomarkers was tested by a likelihood-ratio test [42].

For the meta-analysis, in order to compare effect estimates between studies that analysed biomarker concentrations in quantiles and those that analysed concentrations as continuous variables, we converted effect estimates from the quantile-based analyses into expected estimates if the biomarker had been evaluated as a continuous variable (per SD increase in biomarker concentration); details on the statistical methods and assumptions are included in the Supplementary Material. If only sex-specific effect estimates were reported, those results were pooled using fixed effects models. HRs were calculated using random-effects meta-analysis as proposed by Hedges and Vevea [43]. When multiple multivariable models were included in a publication, we included the effect estimate adjusted for the maximum number of risk factors, but without further adjustment for other biomarkers of vascular injury (if available). If such a model was not available, sensitivity analyses were performed excluding the respective study. Sensitivity analyses by subgroups of study characteristics such as location, fasting vs. non-fasting blood collection and adjustment for covariates were performed (Supplementary Table S5). Heterogeneity between studies was measured by the $\mathrm{I}^{2}$ statistic [44]. Publication bias was not assessed due to the small number of included studies [45]. A two-sided p-value of less than 0.05 denoted statistical significance. SAS version 9.4 (SAS Institute, Cary, North Carolina, USA) for Windows was used to conduct descriptive analyses, imputation of missing data, and Cox regression analyses, while $\mathrm{R}$ statistical software version 3.4.3 was used to conduct the meta-analyses (Metafor $\mathrm{R}$ package), to test for non-linear associations in the Cox regression analyses, and to produce graphs.

\section{Results}

\subsection{Biomarkers of Vascular Injury and Type 2 Diabetes Risk in the EPIC-Heidelberg}

Among 2224 EPIC-Heidelberg participants, 55\% ( $n=1217)$ were women, 163 participants $(7.3 \%)$ developed T2D over a median follow-up of 16 years (p25; p75: 15; 17 years). Participants who developed T2D were more frequently men, had older age, higher prevalence of hypertension, higher BMI, lower educational level and lower levels of physical activity at baseline. Further, these participants had lower high-density lipoprotein (HDL) levels and higher levels of CRP, low-density lipoprotein (LDL), triglycerides, and $\mathrm{HbA1c}$, as well as E-Selectin, P-Selectin, thrombomodulin, GP IIb/IIIa and fibrinogen (Table 1). Median biomarker concentrations for each tertile among women and men are described in Supplementary Table S5.

Table 2 shows associations between biomarkers of vascular injury and T2D risk. In fully adjusted models, E-Selectin was associated with increased T2D risk among study participants in the second, but not the third tertile of E-Selectin concentrations, with HRs of $2.13(95 \% \mathrm{CI} 1.38,3.29)$ and 1.44 $(0.93,2.22)$, respectively. ICAM3 was statistically significantly associated with decreased risk of T2D (HR highest vs. lowest tertile: $0.62(95 \% \mathrm{CI} 0.43,0.91))$. With respect to thrombomodulin, there were no significant differences in diabetes risk across tertiles, despite a non-significant linear trend for an inverse association $(p=0.06)$. When analysing linear trends using continuous values of biomarkers, none were statistically significantly associated with T2D risk. Further adjustments for fibre intake, meat consumption, and coffee intake did not change the results considerably. No statistically significant interactions between any of the biomarkers with age or sex in relation to diabetes risk were observed. There was evidence for non-linearity in the association between E-Selectin levels and T2D risk, as the model which best fitted the data included natural splines with two knots (Supplementary Figure S1), while none of the other biomarkers showed non-linear associations with T2D risk. 
Table 1. Baseline EPIC-Heidelberg subcohort characteristics $(n=2224)$.

\begin{tabular}{|c|c|c|c|}
\hline & $\begin{array}{l}\text { Type } 2 \text { Diabetes Cases } \\
\qquad(n=163)\end{array}$ & $\begin{array}{l}\text { Non-Cases } \\
(n=2061)\end{array}$ & $p$-Value \\
\hline Age at recruitment (years) * & $54.2(47.4 ; 58.7)$ & $50.34(43.0 ; 57.2)$ & 0.09 \\
\hline Women ${ }^{\prime}$ & $62(38.0 \%)$ & $1155(56.0 \%)$ & $<0.001$ \\
\hline Hypertension (yes) $\dagger$ & $94(57.7 \%)$ & $700(34.0 \%)$ & $<0.001$ \\
\hline BMI $\left(\mathrm{kg} / \mathrm{m}^{2}\right)^{*}$ & $29.2(27.6 ; 31.8)$ & $24.9(22.5 ; 27.6)$ & 0.05 \\
\hline Height $(\mathrm{cm}) *$ & $170.2(163.6 ; 176.0)$ & $169.0(162.8 ; 175.7)$ & 0.25 \\
\hline Weight $(\mathrm{kg}) *$ & $85.3(77.0 ; 94.0)$ & $72.2(63.0 ; 82.0)$ & 0.33 \\
\hline Waist circumference $(\mathrm{cm}) *$ & $99.6(92.7 ; 106.0)$ & $86.0(76.0 ; 94.5)$ & 0.10 \\
\hline Alcohol intake at baseline ( $\mathrm{g} /$ day $)^{*}$ & $11.1(3.1 ; 24.5)$ & $10.3(2.9 ; 24.4)$ & $<0.01$ \\
\hline Education level $t$ & & & $<0.001$ \\
\hline Primary School t & $67(41.1 \%)$ & $526(25.5 \%)$ & \\
\hline Secondary School + & $65(39.9 \%)$ & $851(41.3 \%)$ & \\
\hline University Degree $\dagger$ & $31(19.0 \%)$ & $684(33.2 \%)$ & \\
\hline Smoking Status + & & & 0.35 \\
\hline Never $t$ & $68(41.7 \%)$ & $906(44.0 \%)$ & \\
\hline Former, quit $<10 \mathrm{yrs}$ ago $\dagger$ & $29(17.8 \%)$ & $456(22.1 \%)$ & \\
\hline Former, quit $\geq 10 \mathrm{yrs}$ ago $\dagger$ & $23(14.1 \%)$ & $226(11.0 \%)$ & \\
\hline Current $<15$ cigarettes/day $\dagger$ & $21(12.9 \%)$ & $261(12.7 \%)$ & \\
\hline Current $\geq 15$ cigarettes/day $\dagger$ & $22(13.5 \%)$ & $207(10.0 \%)$ & \\
\hline Aspirin use (yes) $\dagger$ & $4(2.5 \%)$ & $57(2.8 \%)$ & 0.81 \\
\hline Antithrombotic drug use (yes) $\dagger$ & $1(0.6 \%)$ & $18(0.9 \%)$ & 0.72 \\
\hline Physical Activity ${ }^{\mathrm{b}}$ (Cambridge index) + & & & 0.03 \\
\hline Inactive/moderately inactive $\dagger$ & $89(54.6 \%)$ & $946(45.9 \%)$ & \\
\hline Moderately active/active $\dagger$ & $74(45.4 \%)$ & $1115(54.1 \%)$ & \\
\hline $\mathrm{CRP}(\mathrm{mg} / \mathrm{l})$ * & $2.0(0.9 ; 3.6)$ & $0.91(0,47 ; 2,29)$ & 0.03 \\
\hline $\mathrm{LDL}(\mathrm{mmol} / \mathrm{l}) *$ & $4.2(3.7 ; 4.9)$ & $3.9(3,3 ; 4,6)$ & 0.90 \\
\hline Triglycerides $(\mathrm{mmol} / \mathrm{l})$ * & $2.2(1.6 ; 3.4)$ & $1.5(1.0 ; 2.2)$ & $<0.001$ \\
\hline $\mathrm{HDL}(\mathrm{mmol} / \mathrm{l}) *$ & $1.2(1.0 ; 1.5)$ & $1.4(1.2 ; 1.8)$ & $<0.01$ \\
\hline Total Cholesterol $(\mathrm{mmol} / \mathrm{l})$ * & $5.9(5.4 ; 6.9)$ & $5.8(5.1 ; 6.5)$ & 0.72 \\
\hline $\mathrm{HbA}_{1 \mathrm{c}}(\mathrm{mmol} / \mathrm{mol}, \%)^{*}$ & $38.0(35.0 ; 41.0), 5.6 \%(5.4 \% ; 5.9 \%)$ & $34.0(32.0 ; 36.0), 5.3 \%(5.1 \% ; 5.4 \%)$ & $<0.001$ \\
\hline E-Selectin $(\mathrm{ng} / \mathrm{mL})^{*}$ & $11.4(8.9 ; 14.9)$ & $9.6(6.9 ; 13.2)$ & 0.32 \\
\hline P-Selectin $(\mathrm{ng} / \mathrm{mL})$ * & $29.6(24.0 ; 38.0)$ & $27.2(21.5 ; 33.9)$ & 0.08 \\
\hline ICAM3 $(\mathrm{ng} / \mathrm{mL})$ * & $0.42(0.36 ; 0.55)$ & $0.44(0.36 ; 0.55)$ & $<0.01$ \\
\hline Thrombomodulin $(\mathrm{ng} / \mathrm{mL})$ * & $3.0(2.6 ; 3.5)$ & $2.9(2.4 ; 3.38)$ & 0.32 \\
\hline Thrombopoietin $(\mathrm{pg} / \mathrm{mL})$ * & $334.2(294.4 ; 398.4)$ & $342.8(287.6 ; 409.8)$ & $<0.001$ \\
\hline Glycoprotein IIb/IIIIa (ng/mL) * & $402.9(324.8 ; 525.6)$ & $382.1(313.4 ; 490.7)$ & 0.84 \\
\hline Fibrinogen $(\mu \mathrm{g} / \mathrm{mL})^{*}$ & $3,998(3643 ; 4471)$ & $3,731(3350 ; 4204)$ & 0.32 \\
\hline
\end{tabular}

Data presented as * median (p25; p75) for continuous variables, and tn (\%) for categorical variables. Abbreviations: CRP C-reactive protein, LDL low-density lipoprotein, HDL high-density lipoprotein, $\mathrm{HbA}_{1 \mathrm{c}}$ glycated haemoglobin, and ICAM3 intercellular adhesion molecule 3. $p$-values for the differences between means (continuous) and frequencies (categorical) of described parameters between participants who developed T2D and who did not develop T2D. b Physical activity measured through the Cambridge index and categorized into inactive/moderately inactive vs. moderately active/active. 
Table 2. Associations between biomarkers of vascular risk and type 2 diabetes risk in the EPIC-Heidelberg.

\begin{tabular}{|c|c|c|c|c|c|}
\hline & \multirow[b]{2}{*}{ Case Number } & \multicolumn{2}{|c|}{ Model 1} & \multicolumn{2}{|c|}{ Model 2} \\
\hline & & HR (95\%CI) & $p$-Value & HR (95\%CI) & $p$-Value \\
\hline \multicolumn{6}{|l|}{ E-Selectin (ng/mL) } \\
\hline Tertile 1 & 32 & Ref. & & Ref. & \\
\hline Tertile 2 & 63 & $2.05(1.34,3.14)$ & $<0.01$ & $2.13(1.38,3.29)$ & $<0.01$ \\
\hline Tertile 3 & 67 & $2.14(1.40,3.26)$ & $<0.001$ & $1.44(0.93,2.22)$ & 0.10 \\
\hline Continuous (per SD) & 162 & $1.39(1.17,1.64)$ & $<0.001$ & $1.05(0.90,1.24)$ & 0.54 \\
\hline \multicolumn{6}{|l|}{ P-Selectin (ng/mL) } \\
\hline Tertile 1 & 46 & Ref. & & Ref. & \\
\hline Tertile 2 & 51 & $1.08(0.72,1.61)$ & 0.71 & $0.82(0.55,1.24)$ & 0.36 \\
\hline Tertile 3 & 65 & $1.37(0.94,2.00)$ & 0.10 & $0.81(0.54,1.21)$ & 0.30 \\
\hline Continuous (per SD) & 162 & $1.20(1.02,1.42)$ & 0.03 & $0.95(0.81,1.12)$ & 0.57 \\
\hline \multicolumn{6}{|l|}{ ICAM3 (ng/mL) } \\
\hline Tertile 1 & 66 & Ref. & & Ref. & \\
\hline Tertile 2 & 46 & $0.67(0.46,0.98)$ & 0.04 & $0.66(0.45,0.98)$ & 0.04 \\
\hline Tertile 3 & 50 & $0.74(0.51,1.07)$ & 0.11 & $0.62(0.43,0.91)$ & 0.01 \\
\hline Continuous (per SD) & 162 & $0.92(0.79,1.08)$ & 0.32 & $0.89(0.76,1.03)$ & 0.13 \\
\hline \multicolumn{6}{|l|}{$\begin{array}{l}\text { Thrombomodulin } \\
\text { (ng/mL) }\end{array}$} \\
\hline Tertile 1 & 49 & Ref. & & Ref. & \\
\hline Tertile 2 & 54 & $1.07(0.73,1.58)$ & 0.73 & $0.93(0.63,1.39)$ & 0.74 \\
\hline Tertile 3 & 59 & $1.12(0.76,1.64)$ & 0.56 & $0.94(0.63,1.40)$ & 0.74 \\
\hline Continuous (per SD) & 162 & $0.97(0.82,1.14)$ & 0.69 & $0.84(0.71,1.00)$ & 0.06 \\
\hline \multicolumn{6}{|l|}{$\begin{array}{c}\text { Thrombopoietin } \\
(\mathrm{pg} / \mathrm{mL})\end{array}$} \\
\hline Tertile 1 & 54 & Ref. & & Ref. & \\
\hline Tertile 2 & 60 & $1.13(0.78,1.63)$ & 0.51 & $1.06(0.72,1.54)$ & 0.78 \\
\hline Tertile 3 & 49 & $0.86(0.59,1.27)$ & 0.45 & $0.92(0.61,1.37)$ & 0.67 \\
\hline Continuous (per SD) & 163 & $0.91(0.78,1.07)$ & 0.26 & $0.95(0.81,1.12)$ & 0.56 \\
\hline \multicolumn{6}{|l|}{$\begin{array}{l}\text { Glycoprotein IIb/IIIa } \\
\text { (ng/mL) }\end{array}$} \\
\hline Tertile 1 & 55 & Ref. & & Ref. & \\
\hline Tertile 2 & 51 & $0.93(0.63,1.36)$ & 0.70 & $0.79(0.53,1.18)$ & 0.25 \\
\hline Tertile 3 & 56 & $1.02(0.70,1.47)$ & 0.94 & $0.86(0.59,1.26)$ & 0.43 \\
\hline Continuous (per SD) & 162 & $1.01(0.87,1.18)$ & 0.85 & $0.95(0.82,1.10)$ & 0.51 \\
\hline \multicolumn{6}{|l|}{ Fibrinogen $(\mu \mathrm{g} / \mathrm{mL})$} \\
\hline Tertile 1 & 33 & Ref. & & Ref. & \\
\hline Tertile 2 & 45 & $1.31(0.84,2.06)$ & 0.23 & $0.96(0.60,1.52)$ & 0.86 \\
\hline Tertile 3 & 85 & $2.46(1.63,3.69)$ & $<0.001$ & $1.05(0.67,1.64)$ & 0.82 \\
\hline Continuous (per SD) & 163 & $1.46(1.25,1.71)$ & $<0.001$ & $1.02(0.85,1.22)$ & 0.82 \\
\hline
\end{tabular}

Model 1 adjusted for age and sex. Model 2 additionally adjusted for height $(\mathrm{m})$, waist circumference $(\mathrm{cm})$, alcohol consumption (g/day in the past year), smoking status (never, past quit $\geq 10$ years ago, past quit $<10$ years ago, current $<15$ cigarettes/day, current $\geq 15$ cigarettes/day), physical activity (Cambridge index), education level (primary, secondary and university),

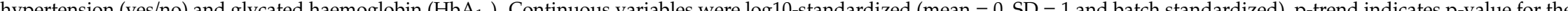
quantitative (log10-linear, standardized) effect: $\mathrm{HR}$ indicates hazard ratio, $\mathrm{CI}$ confidence interval, and ICAM3 intercellular adhesion molecule 3 . 
Diabetes risk prediction analyses showed an AUC of $0.840(95 \%$ CI $0.809,0.870)$ for the basic model (i) (without any vascular injury biomarker). There was statistically significant improvement in the prediction upon addition of (ii) E-Selectin, ICAM3 and thrombomodulin, AUC 0.842 (95\%CI 0.811, $0.874), p=0.02$. When (iii) adding all seven biomarkers to the model, AUC 0.844 (95\%CI 0.812, 0.875), $p=0.15$, there was no significant increase in the prediction ability, in comparison to the basic model (i).

\subsection{Systematic Review and Meta-Analysis}

We identified 2720 unique articles in our initial database searches (Figure 1). After title, abstract and full-text screening, 22 eligible articles that analysed the associations between vascular injury biomarkers and risk of T2D in population-based studies were identified. No relevant abstracts without full-text were found. One article was excluded because despite data availability, the association between fibrinogen and T2D risk was not assessed [46], two articles did not analyse any of the biomarkers of interest [47,48], one article did not report the single effect of fibrinogen, but only its effect combined with CRP [49], and individual findings from the MONICA/KORA [6,50], ARIC [51,52], and Women Health's Initiative $[27,53]$ studies were each reported in two publications, while another study reported change in fibrinogen, and not baseline fibrinogen concentration, in relation to T2D [54]. In case of repeated publications, the one with the higher case number and/or more complete model adjustment was included. Overall 15 studies, including the present one, were included in the systematic review and meta-analysis (Tables 3 and 4). The majority of the studies included populations from the USA or Europe who were middle-aged (mean age range: $32 ; 64$ ), and follow-up durations ranged from 4 to 16 years. Tables 3 and 4 summarize studies and participants characteristics. All included articles were published in English and after 1999. Studies were of high quality, ranging from 7 to 9 out of 9 stars (Newcastle-Ottawa Scale, Table 4, Supplementary Tables S1 and S2). We selected four nested case-control studies $[5,25,27,28]$, seven cohort studies [4,29,52,55-58], one subset of a cohort study [30], and two case-cohort studies [6,24].

Eight studies investigated the relationship between E-Selectin and type 2 diabetes risk [4-6,25,27-30]. All studies reported higher E-Selectin concentrations to be associated with higher T2D risk, with continuous multivariable-adjusted effect estimates ranging from 1.18 (95\%CI 0.90,1.55) [29] to 1.58 $(95 \%$ CI $1.45,1.73)$ [27]. All three studies $[4,30,56]$ that investigated the association between P-Selectin and type 2 diabetes risk found no significant relationships. In the single prior study on thrombomodulin, a borderline inverse association $(0.79(95 \% \mathrm{CI} 0.62,1.00)$ per SD increase in concentration, $p=0.047)$ was reported [24]. In the 5 studies on fibrinogen and T2D, effect estimates ranged from $0.91(95 \% \mathrm{CI}$ $0.75,1.11)[56]$ to $7.18(95 \% \mathrm{CI} 1.63,31.53)$ [58] in the continuous analyses, and from $1.0(95 \% \mathrm{CI} 0.8$, 1.3) [57] to $1.2(95 \% \mathrm{CI} 1.0,1.5)$ [52] when comparing highest to lowest fibrinogen quartiles. No studies on ICAM3, thrombopoietin, and GP IIb/IIIa in relation to type 2 diabetes risk were found.

\subsection{Meta-Analysis}

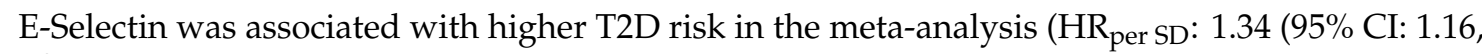
$1.54) ; \mathrm{I}^{2}=63 \% ; n=9$ studies) (Figure 2 ). Analyses using the less adjusted models showed similar results (exclusion of one study that did not report age-and -sex adjusted models [30]) (HR per SD: 1.45 (95\% CI: $1.25,1.70) ; \mathrm{I}^{2}=78 \% ; n=8$ studies). As one study on E-Selectin [4] did not report a multivariable model only adjusted for epidemiological covariates (only also adjusted for other biomarkers), we performed sensitivity analyses excluding this study. Results changed only marginally ( $\mathrm{HR}_{\text {per SD }} 1.32(1.14,1.54)$, $\mathrm{I}^{2}=67 \%$, Supplementary Figure S2). Also, exclusion of the present study only led to marginal changes (Supplementary Figure S3). When including in the meta-analysis only those reported estimates from models with the most comprehensive adjustment (i.e., with additional biomarkers beyond epidemiological covariates) from each study, results changed only slightly. At the same time, there was a tendency for weaker associations in studies with an overall higher degree of adjustment compared to those with less comprehensive adjustment (Supplementary Table S6). Sensitivity analyses on E-Selectin selecting Herder et al. [50] instead of Thorand et al. [6] (same study population) showed no material 
differences in the results (Supplementary Figure S4). Similarly, no material changes were observed when using two estimates for men and women from Thorand et al. [6], instead of pooling them before entering in the meta-analysis (Supplementary Figure S5). The association between thrombomodulin and type 2 diabetes risk was assessed in one other study [24] besides ours. A significant inverse association was observed between thrombomodulin and T2D risk in the meta-analysis ( $\mathrm{HR}_{\text {per }} \mathrm{SD}$ : 0.82 $\left.(0.71,0.95), \mathrm{I}^{2}=0 \%\right)$. Although the previous study reported non-transformed estimates, and we used $\log -10$ transformed estimates, this transformation changed our results only marginally. No statistically significant association was observed between P-Selectin $\left(\mathrm{HR}_{\mathrm{per} \mathrm{SD}} 0.98(0.89,1.07) ; \mathrm{I}^{2}=0 \%, n=4\right.$ studies) or fibrinogen and T2D risk ( $\mathrm{HR}_{\text {per SD }}: 1.01$ (95\%CI 0.91, 1.13); $\mathrm{I}^{2}=52 \%, n=6$ studies) in the meta-analysis (Figure 2).

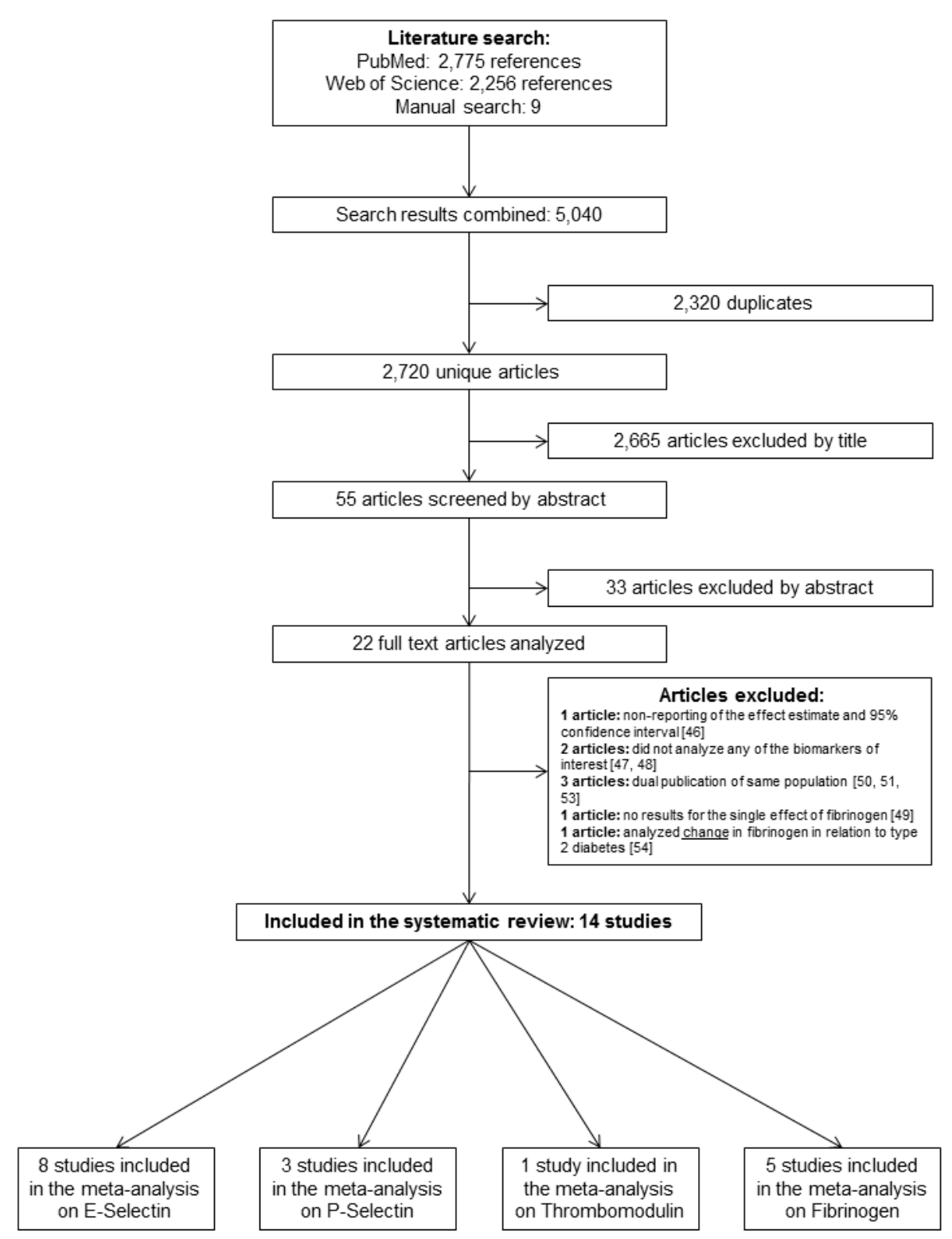

Figure 1. Flowchart illustrating selection of studies for the systematic review and meta-analysis. The present study using EPIC-Heidelberg data is not included. 
Table 3. Characteristics of the studies included in the systematic review on vascular injury biomarkers and type 2 diabetes risk.

\begin{tabular}{|c|c|c|c|c|c|c|c|c|c|c|c|c|c|c|}
\hline \multirow{3}{*}{$\begin{array}{l}\text { First } \\
\text { Author, } \\
\text { Year }\end{array}$} & \multirow{3}{*}{ Study } & \multirow{3}{*}{ Ethnicity (\%) } & \multicolumn{2}{|c|}{ Age (Years, Mean) } & \multicolumn{2}{|c|}{$\begin{array}{c}\text { BMI } \\
\left(\mathrm{kg} / \mathrm{m}^{2} \text {, Mean }\right)\end{array}$} & \multicolumn{2}{|c|}{$\begin{array}{c}\text { Hypertension } \\
\text { (\% Yes) }\end{array}$} & \multicolumn{2}{|c|}{$\begin{array}{c}\text { Current Smokers } \\
\text { (\% Yes) }\end{array}$} & \multirow{3}{*}{$\begin{array}{c}\text { Participants } \\
\text { without Type } \\
2 \text { Diabetes at } \\
\text { Baseline }\end{array}$} & \multirow{3}{*}{$\begin{array}{l}\text { Incident } \\
\text { Type 2 } \\
\text { Diabetes } \\
\text { Cases }\end{array}$} & \multirow{3}{*}{$\begin{array}{l}\text { Mean } \\
\text { Follow-Up } \\
\text { (Years) }\end{array}$} & \multirow{3}{*}{$\begin{array}{c}\text { Definition of Type } 2 \text { Diabetes } \\
\text { Diagnosis }\end{array}$} \\
\hline & & & \multicolumn{8}{|c|}{ Developed Type 2 Diabetes } & & & & \\
\hline & & & No & Yes & No & Yes & No & Yes & No & Yes & & & & \\
\hline $\begin{array}{l}\text { Duncan, } \\
1999[52]\end{array}$ & ARIC & $\begin{array}{l}\text { W: } 78, \\
\text { AA: } 21\end{array}$ & $\begin{array}{r}54 \mathrm{am} \\
(\mathrm{me}\end{array}$ & $\begin{array}{l}\text { ng all } \\
\text { ian) }\end{array}$ & $\begin{array}{r}26 \mathrm{am} \\
(\mathrm{me}\end{array}$ & $\begin{array}{l}\text { ng all } \\
\text { ian) }\end{array}$ & $30 \mathrm{am}$ & ng all & & ng all & 12,330 & 1335 & 7 & $\begin{array}{l}\text { Reported physician diagnosis, } \\
\text { fasting plasma glucose } \geq 7 \mathrm{mmol} / \mathrm{L}, \\
\text { casual glucose } \geq 11.1 \mathrm{mmol} / \mathrm{L} \text {, or } \\
\text { antidiabetic medication use }\end{array}$ \\
\hline $\begin{array}{l}\text { Festa, } 2002 \\
\quad[55]\end{array}$ & $\begin{array}{l}\text { Insulin Resistance } \\
\text { Atherosclerosis } \\
\text { Study }\end{array}$ & $\begin{array}{l}\text { Cases W: } 37, \\
\text { B: } 28, \text { H: } 35 \\
\text { Non-cases W: } \\
\text { 41, B: 26, H:33 }\end{array}$ & 55 & 56 & 28 & 31 & 30 & 45 & $\mathrm{NA}$ & $\mathrm{NA}$ & 1,047 & 144 & 5.2 & WHO criteria [59] \\
\hline $\begin{array}{l}\text { Krakoff, } \\
2003[25]\end{array}$ & $\begin{array}{l}\text { Longitudinal } \\
\text { health study } \\
\text { in Pima Indians }\end{array}$ & Pima Indians & 32 & 33 & 36 & 36 & $\mathrm{NA}$ & NA & $\mathrm{NA}$ & NA & 142 & 71 & $\begin{array}{l}4.6(\text { cases }) \\
6.8 \text { (controls) }\end{array}$ & WHO criteria [59] \\
\hline $\begin{array}{l}\text { Meigs, } \\
2004[5]\end{array}$ & $\begin{array}{l}\text { Nurses' Health } \\
\text { Study }\end{array}$ & $\begin{array}{c}\text { W: } 95, \\
\text { Non-white: } 5\end{array}$ & 56 & 56 & 26 & 30 & $\mathrm{NA}$ & NA & 13 & 14 & 1,446 & 700 & 10 & $\begin{array}{c}\text { Treatment with either insulin or an } \\
\text { oral hypoglycaemic agent, at least } \\
1 \text { classic symptom of diabetes plus } \\
\text { an elevated plasma glucose level, } \\
\text { or an elevated plasma glucose } \\
\text { level on } 2 \text { occasions. Elevated } \\
\text { plasma glucose was defined as at } \\
\text { least } 140 \mathrm{mg} / \mathrm{dL}(\geq 7.8 \mathrm{mmol} / \mathrm{L}) \\
\text { fasting, at least } 200 \mathrm{mg} / \mathrm{dL}(\geq 11.1 \\
\mathrm{mmol} / \mathrm{L}) 2 \mathrm{~h} \text { after an oral glucose } \\
\text { tolerance test for cases diagnosed } \\
\text { before } 1998 ; \text { for cases diagnosed in } \\
1998 \text { and later, the fasting plasma } \\
\text { glucose threshold was } 126 \mathrm{mg} / \mathrm{dL} \\
(\geq 7 \mathrm{mmol} / \mathrm{L}) .\end{array}$ \\
\hline $\begin{array}{l}\text { Thorand, } \\
2006[6]\end{array}$ & MONICA/KORA & White & $\begin{array}{l}51.88^{1 /} / \\
51.7 \stackrel{9}{q}\end{array}$ & $\begin{array}{l}56.10^{x} / \\
56.29\end{array}$ & $\begin{array}{l}27.1 \sigma^{7 /} \\
26.4 \%\end{array}$ & $\begin{array}{l}29.7 \sigma^{71} \\
30.9 \%\end{array}$ & $\begin{array}{l}43.90^{7 /} \\
35.9 \%\end{array}$ & $\begin{array}{l}65.80^{7} / \\
69.4 \%\end{array}$ & $\begin{array}{l}29.40^{x /} \\
19.29\end{array}$ & $\begin{array}{l}35.10^{7 /} \\
15.3 \%\end{array}$ & 2,244 & 532 & 12 & $\begin{array}{c}\text { Physician diagnosis of diabetes, } \\
\text { on participants who self-reported } \\
\text { diabetes }\end{array}$ \\
\hline $\begin{array}{l}\text { Song, } 2007 \\
\quad[27]\end{array}$ & $\begin{array}{l}\text { Women's Health } \\
\text { Initiative } \\
\text { Observational } \\
\text { Study }\end{array}$ & $\begin{array}{l}\text { W: } 51, \\
\text { B: } 29, \\
\text { H: } 12, \\
\text { A: } 8\end{array}$ & $\begin{array}{l}\text { W:64, } \\
\text { B:61, } \\
\text { H:60, } \\
\text { A: } 64\end{array}$ & $\begin{array}{l}\text { W:64, } \\
\text { B:61, } \\
\text { H:60, } \\
\text { A:64 }\end{array}$ & $\begin{array}{l}\text { W:26, } \\
\text { B:30, } \\
\text { H:28, } \\
\text { A:24 }\end{array}$ & $\begin{array}{l}\text { W:33, } \\
\text { B: } 34, \\
\text { H:31, } \\
\text { A: } 27\end{array}$ & $\begin{array}{l}\text { W:6, } \\
\text { B:11, } \\
H: 3, \\
A: 4\end{array}$ & $\begin{array}{l}\text { W: 7, } \\
\text { B:11, } \\
\text { H:8, } \\
\text { A:3 }\end{array}$ & $\mathrm{NA}$ & $\mathrm{NA}$ & 3,782 & 1584 & 5.5 & $\begin{array}{l}\text { Self-report of first-time use of } \\
\text { hypoglycaemic medication (oral } \\
\text { hypoglycaemic agents or insulin) }\end{array}$ \\
\hline $\begin{array}{l}\text { Thorand, } \\
2007 \text { [24] }\end{array}$ & MONICA/KORA & White & 51 & 56 & 27 & 30 & 40 & 67 & 23 & 27 & 1,204 & 224 & 8 & $\begin{array}{l}\text { Self-report of diabetes diagnosed } \\
\text { by a physician or intake of } \\
\text { antidiabetic medication was } \\
\text { validated by physician contact or } \\
\text { medical chart review }\end{array}$ \\
\hline $\begin{array}{l}\text { Stranges, } \\
2008[28]\end{array}$ & $\begin{array}{l}\text { Western New } \\
\text { York Study }\end{array}$ & W: 90 & 60 & 58 & 29 & 32 & 35 & 56 & 11 & 20 & 219 & 61 & 5.9 & $\begin{array}{l}\text { Fasting glucose }>125 \mathrm{mg} / \mathrm{dL} \text { or } \\
\text { antidiabetic medication intake }\end{array}$ \\
\hline $\begin{array}{r}\text { Bertoni, } \\
2010[57]\end{array}$ & MESA & $\begin{array}{l}\text { W: } 42, \\
\text { C:12, } \\
\text { B: } \\
26, \mathrm{H}: 21\end{array}$ & $\begin{array}{l}\text { W: } 62, \mathrm{C}: \\
\text { H: } 6 \text { (al } \\
\text { cases and }\end{array}$ & $\begin{array}{l}\text { l,AA: 62, } \\
\text { ong all, } \\
\text { on-cases) }\end{array}$ & $\begin{array}{r}\text { W: } 28, \mathrm{C} \\
\text { H:29 (a } \\
\text { cases and }\end{array}$ & $\begin{array}{l}\text { 4, AA:30, } \\
\text { ong all, } \\
\text { on-cases) }\end{array}$ & $\begin{array}{l}\text { W: 37, C: } \\
\text { H:37 (a } \\
\text { cases and }\end{array}$ & $\begin{array}{l}\text { 4, AA: } 55 \text {, } \\
\text { ong all, } \\
\text { on-cases) }\end{array}$ & $\begin{array}{r}\text { W: } 11, \\
\text { (amo }\end{array}$ & $\begin{array}{l}\text { A: } 18, \text { H: } 14 \\
\text { cases and } \\
\text { ases) }\end{array}$ & 5,571 & 410 & 4.7 & $\begin{array}{l}\text { Use of diabetes drugs or glucose } \geq \\
\qquad 7 \mathrm{mmol} / \mathrm{L}\end{array}$ \\
\hline
\end{tabular}


Table 3. Cont

\begin{tabular}{|c|c|c|c|c|c|c|c|c|c|c|c|c|c|c|}
\hline \multirow{3}{*}{$\begin{array}{l}\text { First } \\
\text { Author, } \\
\text { Year }\end{array}$} & \multirow{3}{*}{ Study } & \multirow{3}{*}{ Ethnicity (\%) } & \multicolumn{2}{|c|}{ Age (Years, Mean) } & \multicolumn{2}{|c|}{$\begin{array}{c}\text { BMI } \\
\left(\mathrm{kg} / \mathrm{m}^{2}, \text { Mean }\right)\end{array}$} & \multicolumn{2}{|c|}{$\begin{array}{c}\text { Hypertension } \\
\text { (\% Yes) }\end{array}$} & \multicolumn{2}{|c|}{$\begin{array}{c}\text { Current Smokers } \\
\text { (\% Yes) }\end{array}$} & \multirow{3}{*}{$\begin{array}{c}\text { Participants } \\
\text { without Type } \\
\text { 2 Diabetes at } \\
\text { Baseline }\end{array}$} & \multirow{3}{*}{$\begin{array}{c}\text { Incident } \\
\text { Type 2 } \\
\text { Diabetes } \\
\text { Cases }\end{array}$} & \multirow{3}{*}{$\begin{array}{l}\text { Mean } \\
\text { Follow-Up } \\
\text { (Years) }\end{array}$} & \multirow{3}{*}{$\begin{array}{c}\text { Definition of Type } 2 \text { Diabetes } \\
\text { Diagnosis }\end{array}$} \\
\hline & & & \multicolumn{8}{|c|}{ Developed Type 2 Diabetes } & & & & \\
\hline & & & No & Yes & No & Yes & No & Yes & No & Yes & & & & \\
\hline $\begin{array}{l}\text { Dallmeier, } \\
2012[56]\end{array}$ & $\begin{array}{l}\text { Framingham } \\
\text { Heart Study }\end{array}$ & $\begin{array}{l}\text { W: } 93, \\
\text { AA:3, } \\
\text { H: } 2, \\
\text { A: } 2\end{array}$ & 59 & 62 & 28 & 32 & 37 & 68 & 12 & 11 & 2,638 & 162 & 6.6 & $\begin{array}{l}\text { Fasting glucose } \geq 126 \mathrm{mg} / \mathrm{dL} \text { or the } \\
\text { use of insulin or oral } \\
\text { hypoglycaemic medication }\end{array}$ \\
\hline $\begin{array}{c}\text { Julia, 2014 } \\
\text { [29] }\end{array}$ & SU. VI. MAX 2 & French & 51 & 52 & 24.38 & 28.24 & NA & NA & NA & NA & 1,263 & 82 & 13 & $\begin{array}{l}\text { Fasting glucose } \geq 126 \mathrm{mg} / \mathrm{dL} \text { or } \\
\text { use of antidiabetic medication }\end{array}$ \\
\hline $\begin{array}{l}\text { Odegaard, } \\
2016[30]\end{array}$ & CARDIA & B: 44 & $\begin{array}{l}\text { P-Selectin } \\
\text { quartiles } \\
\quad\left(1^{\text {st }} \text { to }\right. \\
4^{\text {th) }}: 40 \\
40,40 \\
40\end{array}$ & $\begin{array}{l}\text { E-Selectin } \\
\text { quartiles } \\
\left(1^{\text {st }} \text { to }\right. \\
4^{\text {th) }}: 40 \\
40,40 \\
40\end{array}$ & $\begin{array}{l}\text { P-Selectin } \\
\text { quartiles } \\
\left(1^{\text {st }} \text { to }\right. \\
4^{\text {th) }}: 27 \\
28,29 \\
29\end{array}$ & $\begin{array}{l}\text { E-Selectin } \\
\text { quartiles } \\
\left(1^{\text {st }} \text { to }\right. \\
4^{\text {th) }}: 26 \\
27,29 \\
31\end{array}$ & NA & NA & $\begin{array}{l}\text { P-Selectin } \\
\text { quartiles } \\
\text { (1t to } \\
4^{\text {th) }}: 12 \\
17,18 \\
28\end{array}$ & $\begin{array}{c}\text { E-Selectin } \\
\text { quartiles }\left(1^{\text {st }} \text { to }\right. \\
4^{\text {th) }}: 11,17,21,27\end{array}$ & 2,339 & $\begin{array}{l}222 \text { for } \\
\text { E-Selectin and } \\
220 \text { for } \\
\text { P-Selectin }\end{array}$ & 10 & $\begin{array}{l}\text { Use of diabetes medication, fasting } \\
\text { blood glucose } \geq 7 \mathrm{mmol} / \mathrm{L}(126 \\
\mathrm{mg} / \mathrm{dL} \mathrm{g}, 2 \mathrm{~h} \text { post-challenge glucose } \\
\geq 11.1 \mathrm{mmol} / \mathrm{L}(200 \mathrm{mg} / \mathrm{dL}) \text {, or a } \\
\text { HbA1c } \geq 6.5 \%(48 \mathrm{mmol} / \mathrm{mol})\end{array}$ \\
\hline $\begin{array}{l}\text { Pankow, } \\
2016[4]\end{array}$ & MESA & $\begin{array}{l}\text { W: } 38, \\
\text { B: } 28, \\
\text { H: } 23, \\
\text { A: } 11\end{array}$ & $\begin{array}{c}\text { P-Selectin } \\
\text { quartiles } \\
\text { (1t }^{\text {st }} \text { to } \\
\left.4^{\text {th }}\right): 61 \\
63,63 \\
62 \\
62\end{array}$ & $\begin{array}{l}\text { E-Selectin } \\
\text { quartiles } \\
\left(1^{\text {st }} \text { to }\right. \\
\left.4^{\text {th) }}\right): 59 \\
59,59 \\
58\end{array}$ & $\begin{array}{l}\text { P-Selectin } \\
\text { quartiles } \\
\left(1^{\text {st }} \text { to }\right. \\
4^{\text {th) }}: 27 \\
27,27 \\
28\end{array}$ & $\begin{array}{c}\text { E-Selectin } \\
\text { quartiles } \\
\left(1^{\text {st }} \text { to }\right. \\
\left.4^{\text {th) }}\right): 61 \\
63.63 . \\
62 \\
62\end{array}$ & NA & NA & NA & NA & $\begin{array}{l}\text { E-Selectin: } \\
\text { 826; } \\
\text { P-Selectin: } \\
\text { 1894 }\end{array}$ & $\begin{array}{l}\text { E-Selectin: } \\
\text { 107; } \\
\text { P-Selectin: } \\
\text { 184 }\end{array}$ & 10 & $\begin{array}{l}\text { Use of insulin or oral diabetes } \\
\text { medication or fasting glucose } \geq 7 \\
\mathrm{mmol} / \mathrm{L}(126 \mathrm{mg} / \mathrm{dL})\end{array}$ \\
\hline $\begin{array}{l}\text { De Simone, } \\
2017[58]\end{array}$ & $\begin{array}{l}\text { Strong Heart } \\
\text { Study }\end{array}$ & $\begin{array}{l}\text { American } \\
\text { Indians }\end{array}$ & 44 & 47 & 30 & 35 & NA & NA & NA & NA & 2,887 & 297 & 4 & $\begin{array}{l}\text { Fasting glucose } \geq 126 \mathrm{mg} / \mathrm{dL} \text { or the } \\
\text { use of antidiabetic medication }\end{array}$ \\
\hline $\begin{array}{l}\text { Pletsch-Borb } \\
2019\end{array}$ & PIC-Heidelberg & White & $\begin{array}{c}50 \\
\text { (median) }\end{array}$ & $\begin{array}{c}54 \\
\text { (median) }\end{array}$ & 25 & 29 & 34 & 58 & 23 & 26 & 2,224 & 163 & $\begin{array}{c}16 \\
\text { (median) }\end{array}$ & $\begin{array}{l}\text { Physician's diagnosis and medical } \\
\text { records review }\end{array}$ \\
\hline
\end{tabular}

Symbols o represent men and pwomen. Abbreviations: BMI body mass index, ARIC: Atherosclerosis Risk in Communities, MONIKA/KORA Multinational monitoring of trends and determinants in cardiovascular disease/"Kooperative Gesundheitsforschung in der Region Augsburg", MESA: Multi-Ethnic Study of Atherosclerosis, SU. VI. MAX 2: Supplémentation en Vitamines et Minéraux AntioXydants, CARDIA: Coronary artery risk development in young adults, EPIC European Prospective Investigation into Cancer and Nutrition, NA not available, $\mathrm{W}$ white, AA African American, B Black, C Chinese, H Hispanic, WHO: world health organization. WHO guidelines incident type 2 diabetes: fasting plasma glucose $\geq 7 \mathrm{mmol} / \mathrm{L}$ or $2 \mathrm{~h}$ plasma glucose $\geq 11.1 \mathrm{mmol} / \mathrm{L}$. 
Table 4. Relationship between plasma markers of endothelial dysfunction and type 2 diabetes risk.

\begin{tabular}{|c|c|c|c|c|c|c|c|c|c|}
\hline $\begin{array}{l}\text { Author, } \\
\text { Year }\end{array}$ & Risk Marker & $\begin{array}{l}\text { Reduced Model for } \\
\text { Type 2 Diabetes Risk } \\
\text { (95\% CI) }\end{array}$ & $\begin{array}{l}\text { Multivariable-Adjusted } \\
\text { Model for Type } 2 \\
\text { Diabetes Risk }(95 \% \mathrm{CI})\end{array}$ & Quantiles & $\begin{array}{l}\text { Reduced Model for Type } \\
2 \text { Diabetes Risk } \\
\text { Continuous (per SD) } \\
\text { (95\% CI) }\end{array}$ & $\begin{array}{c}\text { Multivariable-Adjusted Model for } \\
\text { Type 2 Diabetes Risk } \\
\text { Continuous (per SD) } \\
\text { (95\% CI) }\end{array}$ & $\begin{array}{l}\text { Adjustment for Covariates } \\
\text { (Multivariable-adj. Model) }\end{array}$ & $\begin{array}{l}\text { Study } \\
\text { Quality } \\
\text { (max 9) }\end{array}$ & Study Design \\
\hline $\begin{array}{l}\text { Duncan, } \\
1999[52]\end{array}$ & Fibrinogen & $1.5, p<0.001$ & $1.2(1.0,1.5)$ & quartile & NA & $1.07(1.00,1.15)^{*}$ & $1,2,4,6,10,12,23,25$ & 8 & Cohort \\
\hline $\begin{array}{c}\text { Festa, } \\
2002[55]\end{array}$ & Fibrinogen & NA & $\mathrm{NA}$ & $\mathrm{NA}$ & $1.21(1.01,1.44)$ & $1.02(0.85,1.24)$ & $1,2,4,8,10$ & 8 & Cohort \\
\hline $\begin{array}{l}\text { Krakoff, } \\
2003[25]\end{array}$ & E-Selectin & NA & NA & $\mathrm{NA}$ & $1.12(0.82,1.55)$ & $1.34(0.91,1.99)$ & $1,9,22,23,24,32$ & 7 & $\begin{array}{l}\text { Nested case - } \\
\text { control }\end{array}$ \\
\hline $\begin{array}{l}\text { Meigs, } \\
2004[5]\end{array}$ & E-Selectin & $7.50(5.05,11.14)$ & $4.84(3.06,7.67)$ & Quintile & $1.83(1.37,2.45)^{*}$ & $1.56(1.12,2.18)^{*}$ & $1,6,8,10,11,25,27,30,31$ & 8 & $\begin{array}{c}\text { Nested } \\
\text { case-control }\end{array}$ \\
\hline $\begin{array}{l}\text { Thorand, } \\
2006[6]\end{array}$ & E-Selectin & $\begin{array}{l}3.44(2.46,4.83) \sigma^{7 /} \\
2.79(1.87,4.17) \stackrel{9}{9}\end{array}$ & $\begin{array}{l}2.79(1.91,4.09) \sigma^{7 /} \\
1.72(1.07,2.75) \stackrel{9}{q}\end{array}$ & Tertile & $\begin{array}{c}1.58(1.24,2.02) * \sigma^{7} 1.44 \\
(1.08,1.93) * \frac{9}{*}\end{array}$ & $1.42(1.07,1.88) * \sigma^{7} / 1.18(0.85,1.64) * \%$ & $1,3,8,10,11,12,13,14,20,25,26$ & 9 & Case-cohort \\
\hline $\begin{array}{c}\text { Song, } \\
2007[27]\end{array}$ & E-Selectin & $5.48(4.33,6.94)$ & $3.46(2.56,4.68)$ & Quartile & $1.83(1.70,1.97)$ & $1.58(1.45,1.73)$ & $1,4,5,6,7,8,10,11,12,25,27$ & 8 & $\begin{array}{c}\text { Nested } \\
\text { case-control }\end{array}$ \\
\hline $\begin{array}{l}\text { Thorand, } \\
2007[24]\end{array}$ & Thrombomodulin & NA & NA & NA & $0.92(0.78,1.09)$ & $0.79(0.62,1.00)$ & $1,2,3,8,10,11,12,13,20,25$ & 8 & Case-cohort \\
\hline $\begin{array}{l}\text { Stranges, } \\
2008[28]\end{array}$ & E-Selectin & $3.18(1.32,7.64)$ & $2.77(1.13,6.79)$ & Tertile & $1.63(0.88,3.00)^{*}$ & $1.56(0.84,2.91)^{*}$ & $1,2,5,6,8,23,25$ & 8 & $\begin{array}{c}\text { Nested } \\
\text { case-control }\end{array}$ \\
\hline $\begin{array}{l}\text { Bertoni, } \\
2010[57]\end{array}$ & Fibrinogen & $1.6(1.2,2.1)^{* *}$ & $1.0(0.8,1.3)$ & Quartile & $1.08(0.85,1.36)^{*}$ & $0.92(0.75,1.13)^{*}$ & $1,2,4,6,8,10,11,12,13,15,21,28$ & 7 & Cohort \\
\hline $\begin{array}{l}\text { Dallmeier, } \\
2012[56]\end{array}$ & $\begin{array}{l}\text { P-Selectin and } \\
\text { Fibrinogen }\end{array}$ & NA & NA & NA & $\begin{array}{l}\text { P-Selectin } 1.11(0.94,1.31) \\
\text { and Fibrinogen } 1.24(1.05, \\
1.47)\end{array}$ & $\begin{array}{c}\text { P-Selectin } 0.89(0.74,1.07) \text {, Fibrinogen } \\
0.91(0.75,1.11)\end{array}$ & $1,2,3,8,10,13,18,19,23$ & 8 & Cohort \\
\hline $\begin{array}{l}\text { Julia, } \\
2014[29]\end{array}$ & E-Selectin & $1.18(0.67,2.07)$ & $1.07(0.60,1.93)$ & Tertiles & $1.25(0.96,1.62)$ & $1.18(0.90,1.55)$ & $1,2,8,19,20,23,25,38$ & 8 & Cohort \\
\hline $\begin{array}{l}\text { Odegaard, } \\
2016[30]\end{array}$ & $\begin{array}{l}\text { E-Selectin, } \\
\text { P-Selectin }\end{array}$ & NA & $\begin{array}{l}\text { E-Selectin: } 2.48(1.60, \\
\text { 3.85), P-Selectin: } 1.48 \\
(0.98,2.22)\end{array}$ & Quartiles & NA & $\begin{aligned} \text { E-Selectin } 1.25(0.90,1.75)^{*}, \text { P-Selectin } & 1.09(0.82,1.46)^{*}\end{aligned}$ & $1,2,4,6,10,11,12,25,33$ & 8 & Cohort \\
\hline $\begin{array}{l}\text { Pankow, } \\
2016[4]\end{array}$ & $\begin{array}{l}\text { P-Selectin, } \\
\text { E-Selectin }\end{array}$ & $\begin{array}{l}\text { E-Selectin } 3.76(1.99,7.08), \\
\text { P-Selectin } 1.62(1.06,2.48)\end{array}$ & $\begin{array}{l}\text { E-Selectin } 2.49(1.26,4.93), \\
\text { P-Selectin } 1.14(0.73,1.77)\end{array}$ & quartiles & $\begin{array}{l}\text { E-Selectin } 1.24(1.04,1.49), \\
\text { P-Selectin } 1.06(0.91,1.22)\end{array}$ & $\begin{array}{c}\text { E-Selectin } 1.57(0.95,2.58)^{*} \text {, P-Selectin } \\
1.04(0.89,1.21)\end{array}$ & $\begin{array}{l}\text { Cont.: } 1,2,4,6,8,9,10,12,13,15, \\
22,23,24,26,29 \text {. Quantiles: } 1,2,4, \\
6,8,9,10,12,13,15,21,22 \text { (also } 24, \\
26, \text { and } 29 \text { on E-Selectin analyses) }\end{array}$ & 8 & Cohort \\
\hline $\begin{array}{l}\text { De } \\
\text { Simone, } \\
2017[58]\end{array}$ & Fibrinogen & NA & NA & NA & NA & $7.18(1.63,31.53)$ & $1,2,4,14,34,35,36,37$ & 7 & Cohort \\
\hline $\begin{array}{l}\text { Pletsch-Borl } \\
2019\end{array}$ & rba, All & see Table 2 & see Table 2 & see Table 2 & see Table 2 & see Table 2 & $1,2,8,10,11,12,14,21,22,26$ & 9 & Cohort \\
\hline
\end{tabular}

Abbreviations: CI confidence interval, NA not available, BMI indicates body mass index. Adjustment factors: ${ }^{1}$ age,${ }^{2}$ sex,${ }^{3}$ Survey/cohort, ${ }^{4}$ physical facility/center, ${ }^{5}$ time of blood draw, ${ }^{6}$ race/ethnicity, ${ }^{7}$ duration of follow-up, ${ }^{8} \mathrm{BMI},{ }^{9}$ waist circumference, ${ }^{10}$ smoking status, ${ }^{11}$ alcohol consumption, ${ }^{12}$ physical activity, ${ }^{13}$ SBP, ${ }^{14}$ hypertension, ${ }^{15}$ antihypertensive medication, ${ }^{16}$ total cholesterol, ${ }^{17} \mathrm{LDL},{ }^{18} \mathrm{HDL},{ }^{19}$ triglycerides, ${ }^{20}$ total Cholesterol/HDL cholesterol, ${ }^{21}$ education level, ${ }^{22} \mathrm{HbA1c},{ }^{23}$ fasting glucose, ${ }^{24}$ fasting insulin, ${ }^{25}$ family history of diabetes, ${ }^{26}$ CRP, 27 postmenopausal hormone therapy, ${ }^{28}$ HOMA-IR, ${ }^{29}$ IL6, ${ }^{30}$ fasting status at blood draw, ${ }^{31}$ diet score, ${ }^{32} 2 \mathrm{~h}$ plasma glucose, ${ }^{33}$ carotenoids and tocopherols, ${ }^{34}$ relatedness, ${ }^{35}$ impaired fasting glucose, ${ }^{36}$ body fat, ${ }^{37}$ visceral adiposity, ${ }^{38}$ supplementation group. ${ }^{*}$ Calculated from the quantiles' analyses, as not provided by the original publication. ${ }^{* *}$ Univariate model. Symbols ơ represent men and owomen. Reduced model: age- and sex-adjusted, and in some cases also adjusted for survey, ethnicity, cohort, year of baseline visit, fasting status at blood draw, clinic, smoking. Multivariable-adjusted model: covariates described in column "Adjustment for covariates". 


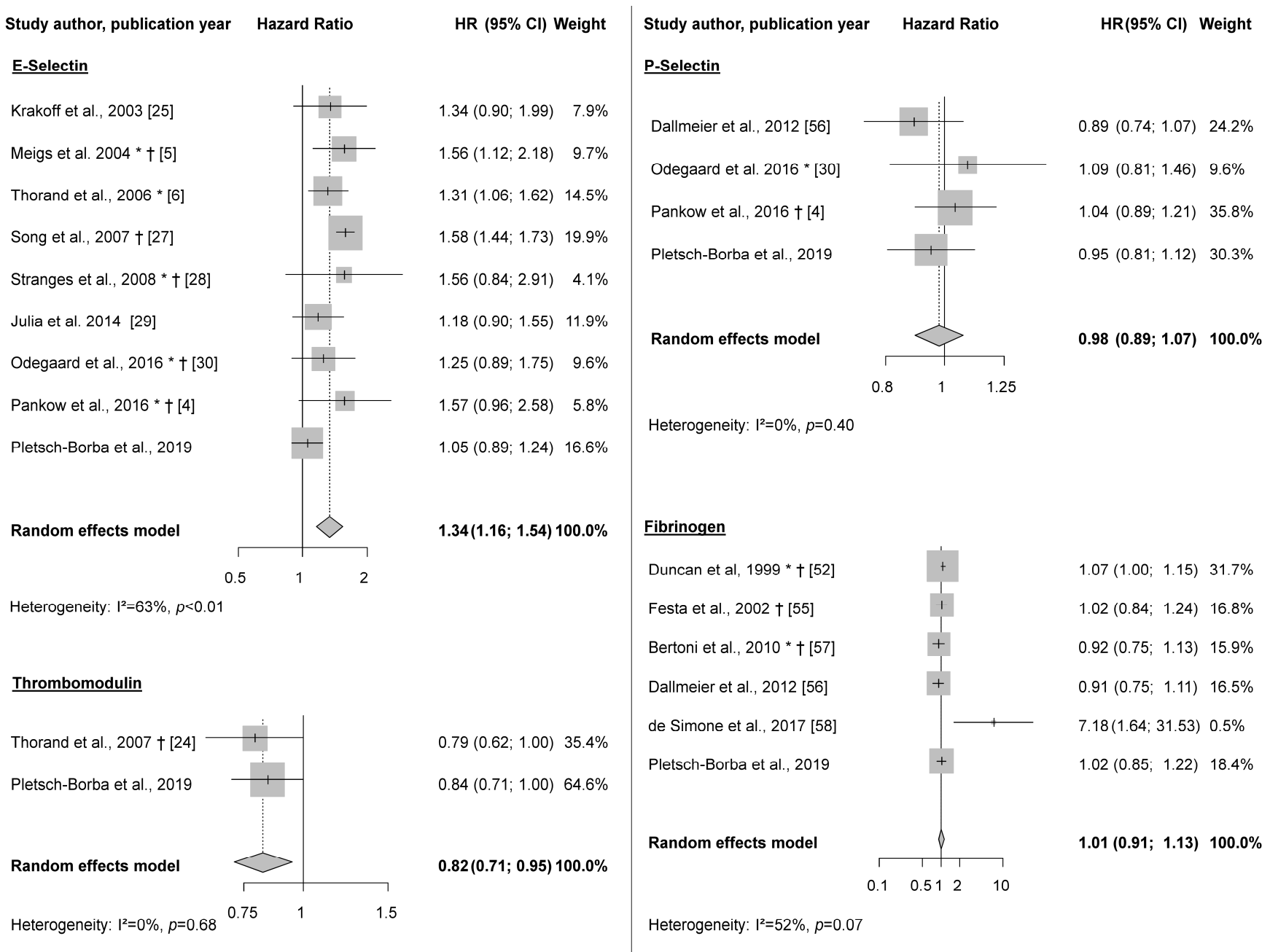

Figure 2. Meta-analysis on biomarkers of vascular injury and type 2 diabetes risk. Random effects meta-analysis on E-Selectin, P-Selectin, Thrombomodulin, and Fibrinogen and type 2 diabetes risk. All effect estimates and confidence intervals derived from the multivariable-adjusted models, as described in Table $4 .{ }^{*}$ Data derived from transformation of quantiles analyses into "per SD", + No log-transformation of the original circulating biomarker concentration performed, except for standardization $($ mean $=0, \mathrm{SD}=1)$. 


\section{Discussion}

In the present analyses of data from the EPIC-Heidelberg, E-Selectin showed a non-linear association with T2D risk, while ICAM3 showed an inverse association with T2D over a median 16 year follow-up period. In the meta-analysis, higher levels of E-Selectin were associated with increased T2D risk, while increased thrombomodulin levels were related to lower T2D risk. The other selected biomarkers showed no association with T2D. No previous studies that explored the association between ICAM3 and T2D were found.

The observation of a positive association between E-Selectin and diabetes risk in our meta-analysis is in line with mechanistic data indicating that E-Selectin, which is expressed on the surface of activated endothelial cells and binds to ligands present on leucocytes, promotes endothelial activation and injury [60]. In a previous cross-sectional study in the EPIC-Heidelberg population, we observed that E-selectin levels are associated with higher BMI, alcohol consumption, LDL-cholesterol, total-cholesterol, and with lower education levels as well as lower HDL-cholesterol [23]. Hence, higher E-Selectin levels may reflect a more unfavourable lifestyle pattern and lower socio-economic status, and co-occur with other cardio-metabolic alterations that lead to T2D. Similarly, whereas in subgroup analyses the positive association between E-Selectin and T2D was present in almost all strata, it was slightly weaker in studies with adjustment for CRP, hypertension, and glucose. Yet, these weaker pooled associations in studies with more comprehensive adjustment should be interpreted carefully, given the lower number of studies.

Our analyses suggested a hat-shaped non-linear association between E-Selectin and T2D in the EPIC-Heidelberg Study. In contrast, a previous meta-analysis by Qiu et al. suggested a non-linear j-shaped association [61], although this association was not statistically significant, and there is no strong indication for non-linearity from prior single studies. Therefore, and due to the lack of an obvious biological explanation for a potential non-linear relationship between E-Selectin levels and T2D risk, we cannot rule out that the non-linear hat-shaped association based on 163 incident cases in our study was due to chance.

We observed an 18\% lower risk of T2D per 1-SD thrombomodulin in our meta-analysis. Besides our study, only one other study investigated thrombomodulin in relation to T2D, and both included only participants from southern Germany [24]. The association observed in the previous study (HR $0.79(0.62,1.00), p=0.047)$ was very similar to the observed in the current one (HR $0.84(0.71,1.00)$, $p=0.06)$, although in the EPIC-Heidelberg it was not statistically significant. The fact that in the MONICA/KORA study a higher number of incident T2D cases occurred may explain this slight difference. Overall, the findings from both studies may support the notion of thrombomodulin exerting anti-coagulative effects [62], and may in turn indicate that vascular injury is implicated in early phases of T2D development, rather than only constituting a late pathophysiological consequence of established T2D. However, further, larger studies are needed to corroborate the present meta-analysis result based on only two cohorts.

The inverse association found in the EPIC-Heidelberg cohort between ICAM3 and T2D risk has not yet been reported from any previous study. ICAM3, a glycoprotein shed by activated leukocytes [15], was demonstrated to be responsible for tethering apoptotic cells to phagocytes, and thus, indicates an increased inflammatory state [14]. Furthermore, ICAM3 has been shown to promote carcinogenesis via pro-inflammatory effects in experimental studies [63]. Results from the present study were unexpected considering that ICAM3 was previously positively associated with prevalent T2D in our cohort, in cross-sectional analyses [23]. Additional studies on this marker are required, and we cannot rule out that the reported association was due to chance, also considering that no significant association between ICAM3 on the continuous scale and T2D was observed.

Three studies $[4,30,56]$, besides the present one, have analysed the association between P-Selectin and T2D risk; all reported null findings. We found no prior studies that examined the association between thrombopoietin and GP IIb/IIIa in relation to T2D risk, and we observed no statistically significant associations between these biomarkers and T2D risk in the current study. Fibrinogen and 
T2D has been investigated in five prior studies [52,55-58], with all but one [58] (in which fibrinogen was positively associated with diabetes risk), reporting no associations. In our study, a positive association was strongly attenuated and no longer statistically significant upon adjustment for confounders. The single positive association reported from the Strong Heart Study may be driven by different ethnicity (American Indians vs. non-Hispanic white from other studies), the younger age of the study population, or the different range of covariates used for statistical adjustment (e.g., body fat instead of BMI) [58].

A potential improvement of T2D risk prediction models upon addition of E-Selectin has been reported by the MONICA/KORA study [50]. The present study suggests further that besides E-Selectin, the inclusion of thrombomodulin and ICAM3 may slightly increase the AUC of prediction models, although further prospective studies on these biomarkers are needed. Moreover, associations between E-Selectin and T2D risk showed heterogeneity across study populations, which may speak against its usefulness for risk stratification. The observation of similar inverse associations with T2D in the two available studies on thrombomodulin (EPIC-Heidelberg, MONICA/KORA) may be related to the fact that both study populations comprised white adults from southern Germany with similar characteristics, and further studies on thrombomodulin and T2D in populations with different ethnic and socio-economic characteristics are needed. In general, it should be noted that the present study and meta-analysis did not have the purpose to generate or validate a T2D prediction model, and that multibiomarker studies with external validation are required to assess whether E-Selectin, ICAM3, and thrombomodulin are potential predictors of absolute T2D risk.

Strengths of our study include the comprehensive set of covariates available for statistical adjustment, including $\mathrm{HbA1}_{\mathrm{c}}$ and CRP. Furthermore, we previously demonstrated that the biomarkers investigated in this study had good one-year reproducibility (Spearman correlations ranges 0.51 to 0.88) [36]. Besides that, we conducted a meta-analysis, in additional to our original research, which synthesizes available data on this topic. Finally, our results on E- and P-Selectin were comparable to the ones reported by a meta-analysis that was performed in parallel to this one [61]. Interestingly, risk estimates for associations between E-Selectin and T2D risk were much stronger in magnitude in this meta-analysis by Qiu et al. [61], which may be due to the fact that E-Selectin concentrations were modelled on the $\mu \mathrm{g} / \mathrm{mL}$ scale. For our analyses, we decided to use the $\mathrm{ng} / \mathrm{mL}$ scale, as E-Selectin concentrations were below $0.1 \mu \mathrm{g} / \mathrm{mL}$ in the majority of blood samples of our study population and others. Thus, risk estimates for E-Selectin in relation to T2D risk from our meta-analysis are lower, but still consistent with those reported by Qiu et al. [61].

Our study also has limitations that must be acknowledged. Although we had a relatively long period of follow-up, we only observed 163 incident cases of T2D in this subcohort of the EPIC-Heidelberg, with limited power for subgroup analyses. The selection of biomarkers for the meta-analysis was opportunistic in that we only included biomarkers which were measured in our own cohort. The relatively low case number may be due to good general health status of the EPIC-Heidelberg participants at baseline. Although this potential "healthy cohort effect" may also have affected our results (towards the null), our results are in agreement with previous studies. Additionally, results from the EPIC-Heidelberg cannot be generalized to non-white populations, and the studies included in the review and meta-analysis were also performed in predominantly white populations, which highlight the need for multi-ethnic studies. Further, these results may not be generalizable to high-risk populations, such as obese individuals. In addition, there may be other interesting biomarkers of vascular injury such as ICAM1 [61] that were not covered by the present meta-analysis, as we focused on biomarkers that had been measured in EPIC-Heidelberg. Associations of vascular injury biomarkers with complications of T2D were out of the scope of this review, and studies on these biomarkers in the context of diabetes complications are needed. Lastly, as in all observational studies, we cannot exclude that the associations we found may be affected by residual confounding, even though we have statistically controlled for a wide range of T2D risk factors. 


\section{Conclusions}

The present findings reiterate that increased E-Selectin, a marker of vascular injury, is associated with increased T2D risk, while higher circulating thrombomodulin levels, indicative of vessel protection, may be related to a decreased risk of developing T2D. Thus, endothelial dysfunction seems to be implicated in early phases of T2D development many years before diagnosis. While E-Selectin and thrombomodulin may be interesting candidate biomarkers for diabetes risk prediction models, further multi-biomarker studies are needed to assess and validate their predictive capacity in a targeted manner.

Supplementary Materials: The following are available online at http://www.mdpi.com/2077-0383/8/12/2075/s1, Systematic Review Search strings, Methods: covariates assessment and statistical analyses, Table S1: Newcastle-Ottawa Quality Assessment, Table S2: Newcastle-Ottawa Quality Assessment for each study, Table S3: Missing data in the EPIC-Heidelberg subcohort, Table S4: Within- and between-batch coefficients of variation, and intra-individual correlation coefficients across vascular injury biomarkers. Table S5: Median concentrations of each biomarker in tertiles of biomarkers in women and men from the EPIC-Heidelberg subcohort, Table S6: Subgroup meta-analyses on the associations between E-Selectin and T2D risk, Figure S1: Non-linear association between log10-E-Selectin concentration (per SD) and risk of type 2 diabetes in the EPIC-Heidelberg, Figure S2: Meta-analysis on E-Selectin excluding one study that only showed a multivariable-adjusted model including other biomarkers of vascular injury, Figure S3: Meta-analysis on E-Selectin and type 2 diabetes risk excluding the present study, EPIC-Heidelberg, Figure S4: Meta-analysis on E-Selectin and type 2 diabetes risk excluding Thorand et al. 2006 and keeping Herder et al. 2011 instead, Figure S5: Meta-analysis on E-Selectin and type 2 diabetes risk, using the two estimates (women and men) provided by Thorand et al.

Author Contributions: T.K. and L.P.-B.: designed the analysis plan; M.G., T.J., C.W., S.A.S., T.K. and R.K.: contributed to the acquisition of data; L.P.-B.: performed statistical analysis; S.G.M. and A.H. contributed to the statistical analyses; L.P.B. wrote the manuscript and had primary responsibility for the final content, T.K. revised it. All authors contributed to the interpretation of data, to reviewing the manuscript critically, and approved its final version. L.P.-B. and T.K. are the study guarantors.

Funding: This research was funded by the World Cancer Research Fund (WCRF UK), as part of the World Cancer Research Fund International grant program, and grant number 2015/1418.

Acknowledgments: The authors thank the laboratory staff of the Division of Cancer Epidemiology, DKFZ, especially Bettina Ehret and Christine Niesik, for their support with the current project.

Conflicts of Interest: The authors declare no conflict of interest. The funders had no role in the design of the study; in the collection, analyses, or interpretation of data; in the writing of the manuscript, or in the decision to publish the results.

\section{References}

1. Guariguata, L.; Whiting, D.R.; Hambleton, I.; Beagley, J.; Linnenkamp, U.; Shaw, J.E. Global estimates of diabetes prevalence for 2013 and projections for 2035. Diabetes Res. Clin. Pract. 2014, 103, 137-149. [CrossRef] [PubMed]

2. Dalys, G.B.D.; Collaborators, H. Global, regional, and national disability-adjusted life-years (DALYs) for 359 diseases and injuries and healthy life expectancy (HALE) for 195 countries and territories, 1990-2017: A systematic analysis for the Global Burden of Disease Study 2017. Lancet 2018, 392, 1859-1922. [CrossRef]

3. Muris, D.M.; Houben, A.J.; Schram, M.T.; Stehouwer, C.D. Microvascular dysfunction is associated with a higher incidence of type 2 diabetes mellitus: A systematic review and meta-analysis. Arter. Thromb. Vasc. Biol. 2012, 32, 3082-3094. [CrossRef] [PubMed]

4. Pankow, J.S.; Decker, P.A.; Berardi, C.; Hanson, N.Q.; Sale, M.; Tang, W.; Kanaya, A.M.; Larson, N.B.; Tsai, M.Y.; Wassel, C.L.; et al. Circulating cellular adhesion molecules and risk of diabetes: The Multi-Ethnic Study of Atherosclerosis (MESA). Diabet. Med. A J. Br. Diabet. Assoc. 2016, 33, 985-991. [CrossRef]

5. Meigs, J.B.; Hu, F.B.; Rifai, N.; Manson, J.E. Biomarkers of endothelial dysfunction and risk of type 2 diabetes mellitus. JAMA 2004, 291, 1978-1986. [CrossRef]

6. Thorand, B.; Baumert, J.; Chambless, L.; Meisinger, C.; Kolb, H.; Doring, A.; Lowel, H.; Koenig, W.; Group, M.K.S. Elevated markers of endothelial dysfunction predict type 2 diabetes mellitus in middle-aged men and women from the general population. Arter. Thromb. Vasc. Biol. 2006, 26, 398-405. [CrossRef]

7. Miles, P.D.; Levisetti, M.; Reichart, D.; Khoursheed, M.; Moossa, A.R.; Olefsky, J.M. Kinetics of insulin action in vivo. Identification of rate-limiting steps. Diabetes 1995, 44, 947-953. [CrossRef]

8. Aird, W.C. Endothelium and haemostasis. Hamostaseologie 2015, 35, 11-16. [CrossRef] 
9. Jones, D.A.; Abbassi, O.; McIntire, L.V.; McEver, R.P.; Smith, C.W. P-selectin mediates neutrophil rolling on histamine-stimulated endothelial cells. Biophys. J. 1993, 65, 1560-1569. [CrossRef]

10. Bonfanti, R.; Furie, B.C.; Furie, B.; Wagner, D.D. PADGEM (GMP140) is a component of Weibel-Palade bodies of human endothelial cells. Blood 1989, 73, 1109-1112. [CrossRef]

11. Stenberg, P.E.; McEver, R.P.; Shuman, M.A.; Jacques, Y.V.; Bainton, D.F. A platelet alpha-granule membrane protein (GMP-140) is expressed on the plasma membrane after activation. J. Cell Biol. 1985, 101, 880-886. [CrossRef] [PubMed]

12. Laubli, H.; Borsig, L. Selectins promote tumor metastasis. Semin. Cancer Biol. 2010, 20, 169-177. [CrossRef] [PubMed]

13. Campanero, M.R.; Sanchez-Mateos, P.; del Pozo, M.A.; Sanchez-Madrid, F. ICAM-3 regulates lymphocyte morphology and integrin-mediated $\mathrm{T}$ cell interaction with endothelial cell and extracellular matrix ligands. J. Cell Biol. 1994, 127, 867-878. [CrossRef] [PubMed]

14. Torr, E.E.; Gardner, D.H.; Thomas, L.; Goodall, D.M.; Bielemeier, A.; Willetts, R.; Griffiths, H.R.; Marshall, L.J.; Devitt, A. Apoptotic cell-derived ICAM-3 promotes both macrophage chemoattraction to and tethering of apoptotic cells. Cell Death Differ. 2012, 19, 671-679. [CrossRef]

15. de Fougerolles, A.R.; Springer, T.A. Intercellular adhesion molecule 3, a third adhesion counter-receptor for lymphocyte function-associated molecule 1 on resting lymphocytes. J. Exp. Med. 1992, 175, 185-190. [CrossRef]

16. Esmon, C.T.; Fukudome, K.; Mather, T.; Bode, W.; Regan, L.M.; Stearns-Kurosawa, D.J.; Kurosawa, S. Inflammation, sepsis, and coagulation. Haematologica 1999, 84, 254-259.

17. Gale, A.J. Continuing education course \#2: Current understanding of hemostasis. Toxicol. Pathol. 2011, 39, 273-280. [CrossRef]

18. Hitchcock, I.S.; Kaushansky, K. Thrombopoietin from beginning to end. Br. J. Haematol. 2014, 165, $259-268$. [CrossRef]

19. Kurokawa, T.; Zheng, Y.W.; Ohkohchi, N. Novel functions of platelets in the liver. J. Gastroenterol. Hepatol. 2016, 31, 745-751. [CrossRef]

20. Lupia, E.; Goffi, A.; Bosco, O.; Montrucchio, G. Thrombopoietin as biomarker and mediator of cardiovascular damage in critical diseases. Mediat. Inflamm. 2012, 2012, 390892. [CrossRef]

21. Kaushansky, K. Thrombopoietin. N. Engl. J. Med. 1998, 339, 746-754. [CrossRef]

22. Jackson, S.P. Arterial thrombosis-insidious, unpredictable and deadly. Nat. Med. 2011, 17, 1423-1436. [CrossRef]

23. Pletsch-Borba, L.; Grafetstätter, M.; Hüsing, A.; González Maldonado, S.; Kloss, M.; Gross, M.L.; Johnson, T.; Sookthai, D.; Bugert, P.; Kaaks, R.; et al. Biomarkers of vascular injury in relation to myocardial infarction risk: A population-based study. Sci. Rep. 2019, 9, 3004. [CrossRef]

24. Thorand, B.; Baumert, J.; Herder, C.; Meisinger, C.; Koenig, W. Soluble thrombomodulin as a predictor of type 2 diabetes: Results from the MONICA/KORA Augsburg case-cohort study, 1984-1998. Diabetologia 2007, 50, 545-548. [CrossRef]

25. Krakoff, J.; Funahashi, T.; Stehouwer, C.D.A.; Schalkwijk, C.G.; Tanaka, S.; Matsuzawa, Y.; Kobes, S.; Tataranni, P.A.; Hanson, R.L.; Knowler, W.C.; et al. Inflammatory markers, adiponectin, and risk of type 2 diabetes in the Pima Indian. Diabetes Care 2003, 26, 1745-1751. [CrossRef]

26. Thorand, B.; Baumert, J.; Doring, A.; Schneider, A.; Chambless, L.; Lowel, H.; Kolb, H.; Koenig, W. Association of cardiovascular risk factors with markers of endothelial dysfunction in middle-aged men and women. Results from the MONICA/KORA Augsburg Study. Thromb. Haemost. 2006, 95, 134-141. [CrossRef]

27. Song, Y.; Manson, J.E.; Tinker, L.; Rifai, N.; Cook, N.R.; Hu, F.B.; Hotamisligil, G.S.; Ridker, P.M.; Rodriguez, B.L.; Margolis, K.L.; et al. Circulating levels of endothelial adhesion molecules and risk of diabetes in an ethnically diverse cohort of women. Diabetes 2007, 56, 1898-1904. [CrossRef]

28. Stranges, S.; Rafalson, L.B.; Dmochowski, J.; Rejman, K.; Tracy, R.P.; Trevisan, M.; Donahue, R.P. Additional contribution of emerging risk factors to the prediction of the risk of type 2 diabetes: Evidence from the Western New York Study. Obesity 2008, 16, 1370-1376. [CrossRef]

29. Julia, C.; Czernichow, S.; Charnaux, N.; Ahluwalia, N.; Andreeva, V.; Touvier, M.; Galan, P.; Fezeu, L. Relationships between adipokines, biomarkers of endothelial function and inflammation and risk of type 2 diabetes. Diabetes Res. Clin. Pract. 2014, 105, 231-238. [CrossRef] 
30. Odegaard, A.O.; Jacobs, D.R., Jr.; Sanchez, O.A.; Goff, D.C., Jr.; Reiner, A.P.; Gross, M.D. Oxidative stress, inflammation, endothelial dysfunction and incidence of type 2 diabetes. Cardiovasc. Diabetol. 2016, 15, 51. [CrossRef]

31. Boeing, H.; Korfmann, A.; Bergmann, M.M. Recruitment procedures of EPIC-Germany. European Investigation into Cancer and Nutrition. Ann. Nutr. Metab. 1999, 43, 205-215. [CrossRef] [PubMed]

32. Boeing, H.; Wahrendorf, J.; Becker, N. EPIC-Germany-A source for studies into diet and risk of chronic diseases. European Investigation into Cancer and Nutrition. Ann. Nutr. Metab. 1999, 43, 195-204. [CrossRef] [PubMed]

33. Graf, M.E.; Sookthai, D.; Johnson, T.; Schübel, R.; González Maldonado, S.; Pletsch-Borba, L.; Katzke, V.; Bugert, P.; Hoffmeister, M.; Kaaks, R.; et al. Pre-diagnostic plasma concentrations of Fibrinogen, sGPIIb/IIIa, sP-selectin, sThrombomodulin, Thrombopoietin in relation to cancer risk: Findings from a large prospective study. Int. J. Cancer 2018. [CrossRef] [PubMed]

34. Grafetstätter, M.; Hüsing, A.; González Maldonado, S.; Sookthai, D.; Johnson, T.; Pletsch-Borba, L.; Katzke, V.A.; Hoffmeister, M.; Bugert, P.; Kaaks, R.; et al. Plasma Fibrinogen and sP-Selectin are Associated with the Risk of Lung Cancer in a Prospective Study. Cancer Epidemiol. Biomark. Prev. 2019, 28, 1221-1227. [CrossRef] [PubMed]

35. Bergmann, M.M.; Bussas, U.; Boeing, H. Follow-up procedures in EPIC-Germany-Data quality aspects. European Prospective Investigation into Cancer and Nutrition. Ann. Nutr. Metab. 1999, 43, 225-234. [CrossRef]

36. Graf, M.E.; Sookthai, D.; Johnson, T.; Schubel, R.; Katzke, V.; Bugert, P.; Hoffmeister, M.; Kaaks, R.; Kühn, T. Biological reproducibility of circulating P-Selectin, Thrombopoietin, GPIIb/IIIa and Thrombomodulin over one year. Clin. Biochem. 2017, 50, 942-946. [CrossRef]

37. Fibrinogen Studies, C.; Wood, A.M.; White, I.; Thompson, S.G.; Lewington, S.; Danesh, J. Regression dilution methods for meta-analysis: Assessing long-term variability in plasma fibrinogen among 27,247 adults in 15 prospective studies. Int. J. Epidemiol. 2006, 35, 1570-1578. [CrossRef]

38. Lazar, C.; Meganck, S.; Taminau, J.; Steenhoff, D.; Coletta, A.; Molter, C.; Weiss-Solis, D.Y.; Duque, R.; Bersini, H.; Nowe, A. Batch effect removal methods for microarray gene expression data integration: A survey. Brief. Bioinform. 2013, 14, 469-490. [CrossRef]

39. Moher, D.; Liberati, A.; Tetzlaff, J.; Altman, D.G.; Group, P. Preferred reporting items for systematic reviews and meta-analyses: The PRISMA statement. PLoS Med. 2009, 6, e1000097. [CrossRef]

40. Wells, G.; Shea, B.; O'connell, D.; Peterson, J.; Welch, V.; Losos, M.; Tugwell, P. The Newcastle-Ottawa Scale (NOS) for Assessing the Quality if Nonrandomized Studies in Meta-Analyses. Available online: http://www.ohri.ca/programs/clinical_epidemiology/oxford.asp (accessed on 24 May 2019).

41. Prentice, R.L.; Self, S.G. Aspects of the use of relative risk models in the design and analysis of cohort studies and prevention trials. Stat. Med. 1988, 7, 275-287. [CrossRef]

42. Pepe, M.S.; Kerr, K.F.; Longton, G.; Wang, Z. Testing for improvement in prediction model performance. Stat. Med. 2013, 32, 1467-1482. [CrossRef] [PubMed]

43. Hedges, L.V.; Vevea, J.L. Fixed- and Random-Effects Models in Meta-Analysis. Psychol. Methods 1998, 3, 486-504. [CrossRef]

44. Higgins, J.P.; Thompson, S.G.; Deeks, J.J.; Altman, D.G. Measuring inconsistency in meta-analyses. BMJ 2003, 327, 557-560. [CrossRef] [PubMed]

45. Egger, M.; Davey Smith, G.; Schneider, M.; Minder, C. Bias in meta-analysis detected by a simple, graphical test. BMJ 1997, 315, 629-634. [CrossRef]

46. Barzilay, J.I.; Abraham, L.; Heckbert, S.R.; Cushman, M.; Kuller, L.H.; Resnick, H.E.; Tracy, R.P. The relation of markers of inflammation to the development of glucose disorders in the elderly: The Cardiovascular Health Study. Diabetes 2001, 50, 2384-2389. [CrossRef] [PubMed]

47. Duncan, B.B.; Schmidt, M.I.; Pankow, J.S.; Ballantyne, C.M.; Couper, D.; Vigo, A.; Hoogeveen, R.; Folsom, A.R.; Heiss, G. Low-grade systemic inflammation and the development of type 2 diabetes-The atherosclerosis risk in communities study. Diabetes 2003, 52, 1799-1805. [CrossRef]

48. Huang, T.; Glass, K.; Zeleznik, O.A.; Kang, J.H.; Ivey, K.L.; Sonawane, A.R.; Birmann, B.M.; Hersh, C.P.; Hu, F.B.; Tworoger, S.S. A Network Analysis of Biomarkers for Type 2 Diabetes. Diabetes 2019, 68, 281-290. [CrossRef] 
49. Koloverou, E.; Panagiotakos, D.B.; Georgousopoulou, E.N.; Chrysohoou, C.; Tousoulis, D.; Stefanadis, C.; Pitsavos, C.; Group, A.S. Single and combined effects of inflammatory markers on 10 year diabetes incidence: The mediating role of adiposity-Results from the ATTICA cohort study. Diabetes Metab. Res. Rev. 2018, 34. [CrossRef]

50. Herder, C.; Baumert, J.; Zierer, A.; Roden, M.; Meisinger, C.; Karakas, M.; Chambless, L.; Rathmann, W.; Peters, A.; Koenig, W.; et al. Immunological and Cardiometabolic Risk Factors in the Prediction of Type 2 Diabetes and Coronary Events: MONICA/KORA Augsburg Case-Cohort Study. PLoS ONE 2011, 6. [CrossRef]

51. Schmidt, M.I.; Duncan, B.B.; Sharrett, A.R.; Lindberg, G.; Savage, P.J.; Offenbacher, S.; Azambuja, M.I.; Tracy, R.P.; Heiss, G. Markers of inflammation and prediction of diabetes mellitus in adults (Atherosclerosis Risk in Communities study): A cohort study. Lancet 1999, 353, 1649-1652. [CrossRef]

52. Duncan, B.B.; Schmidt, M.I.; Offenbacher, S.; Wu, K.K.; Savage, P.J.; Heiss, G. Factor VIII and other hemostasis variables are related to incident diabetes in adults - The Atherosclerosis Risk in Communities (ARIC) study. Diabetes Care 1999, 22, 767-772. [CrossRef] [PubMed]

53. Chao, C.; Song, Y.; Cook, N.; Tseng, C.-H.; Manson, J.E.; Eaton, C.; Margolis, K.L.; Rodriguez, B.; Phillips, L.S.; Tinker, L.F.; et al. The Lack of Utility of Circulating Biomarkers of Inflammation and Endothelial Dysfunction for Type 2 Diabetes Risk Prediction Among Postmenopausal Women The Women's Health Initiative Observational Study. Arch. Intern. Med. 2010, 170, 1557-1565. [CrossRef] [PubMed]

54. Festa, A.; Williams, K.; Tracy, R.P.; Wagenknecht, L.E.; Haffner, S.M. Progression of plasminogen activator inhibitor-1 and fibrinogen levels in relation to incident type 2 diabetes. Circulation 2006, 113, 1753-1759. [CrossRef] [PubMed]

55. Festa, A.; D'Agostino, R.; Tracy, R.P.; Haffner, S.M. Elevated levels of acute-phase proteins and plasminogen activator inhibitor-1 predict the development of type 2 diabetes-The insulin resistance atherosclerosis study. Diabetes 2002, 51, 1131-1137. [CrossRef] [PubMed]

56. Dallmeier, D.; Larson, M.G.; Wang, N.; Fontes, J.D.; Benjamin, E.J.; Fox, C.S. Addition of Inflammatory Biomarkers Did Not Improve Diabetes Prediction in the Community: The Framingham Heart Study. J. Am. Heart Assoc. 2012, 1. [CrossRef]

57. Bertoni, A.G.; Burke, G.L.; Owusu, J.A.; Carnethon, M.R.; Vaidya, D.; Barr, R.G.; Jenny, N.S.; Ouyang, P.; Rotter, J.I. Inflammation and the incidence of type 2 diabetes: The Multi-Ethnic Study of Atherosclerosis (MESA). Diabetes Care 2010, 33, 804-810. [CrossRef]

58. de Simone, G.; Wang, W.; Best, L.G.; Yeh, F.; Izzo, R.; Mancusi, C.; Roman, M.J.; Lee, E.T.; Howard, B.V.; Devereux, R.B. Target organ damage and incident type 2 diabetes mellitus: The Strong Heart Study. Cardiovasc. Diabetol. 2017, 16, 64. [CrossRef]

59. World Health Organization. WHO Expert Committee on Diabetes Mellitus: Second report. World Health Organ. Tech. Rep. Ser. 1985, 646, 1-80.

60. McEver, R.P. Selectins: Lectins that initiate cell adhesion under flow. Curr. Opin. Cell Biol. 2002, 14, 581-586. [CrossRef]

61. Qiu, S.; Cai, X.; Liu, J.; Yang, B.; Zugel, M.; Steinacker, J.M.; Sun, Z.; Schumann, U. Association between circulating cell adhesion molecules and risk of type 2 diabetes: A meta-analysis. Atherosclerosis 2019, 287, 147-154. [CrossRef]

62. Ito, T.; Kakihana, Y.; Maruyama, I. Thrombomodulin as an intravascular safeguard against inflammatory and thrombotic diseases. Expert Opin. Ther. Targets 2016, 20, 151-158. [CrossRef] [PubMed]

63. Shen, W.; Xie, J.; Zhao, S.; Du, R.; Luo, X.; He, H.; Jiang, S.; Hao, N.; Chen, C.; Guo, C.; et al. ICAM3 mediates inflammatory signaling to promote cancer cell stemness. Cancer Lett. 2018, 422, 29-43. [CrossRef] [PubMed]

(C) 2019 by the authors. Licensee MDPI, Basel, Switzerland. This article is an open access article distributed under the terms and conditions of the Creative Commons Attribution (CC BY) license (http://creativecommons.org/licenses/by/4.0/). 